\title{
Conceptual design of a beam line for post-collision extraction and diagnostics at the multi-TeV Compact Linear Collider
}

\author{
Arnaud Ferrari* and Volker Ziemann \\ Department of Physics and Astronomy, Uppsala University, 75121 Uppsala, Sweden \\ Robert B. Appleby and Michael D. Salt \\ University of Manchester, Oxford Road, Manchester M13 9PL, United Kingdom, \\ and The Cockcroft Institute, Daresbury Laboratory, Warrington WA4 4AD, United Kingdom
}

(Received 18 September 2008; published 27 February 2009)

\begin{abstract}
Strong beam-beam effects at the interaction point of a high-energy $e^{+} e^{-}$linear collider such as the Compact Linear Collider (CLIC) lead to an emittance growth for the outgoing beams, as well as to the production of beamstrahlung photons and $e^{+} e^{-}$coherent pairs. In this paper, we present a conceptual design of a $150 \mathrm{~m}$ long post-collision extraction line for the CLIC machine at $3 \mathrm{TeV}$, which separates the various components of the outgoing beam using a vertical magnetic chicane, before transporting them to their respective dump. In addition, detailed studies are performed in order to compute the power losses along the CLIC post-collision line. For the vacuum window at the exit of the post-collision line, we propose a thick $(1.5 \mathrm{~cm})$ layer of carbon-carbon composite, with a thin $(0.2 \mathrm{~mm})$ aluminum leak-tight foil. The stress levels in this exit window are estimated. Finally, we discuss the use of diagnostics along the post-collision line for monitoring and improving the quality of the $e^{+} e^{-}$collisions and, in turn, the luminosity of the CLIC machine.
\end{abstract}

DOI: 10.1103/PhysRevSTAB.12.021001

PACS numbers: 29.27.Eg, 41.75.Ht

\section{INTRODUCTION}

The Compact Linear Collider (CLIC) aims at multi-TeV $e^{+} e^{-}$collisions using the two-beam acceleration technology [1]. In order to keep the length (and thereby the cost) of the machine at a reasonable level, the rf frequency and the accelerating gradient are, respectively, $12 \mathrm{GHz}$ and $100 \mathrm{MV} / \mathrm{m}$. Hence, the bunch spacing is a few $\mathrm{cm}$, which is too short to collide head-on beams, due to the need to avoid parasitic collisions. Instead, the beams must collide with a crossing angle, for which the optimal value is $20 \mathrm{mrad}$ [2]. In order to force colliding bunches to be perfectly aligned (and thus to recover the desired luminosity), crab cavities are used to deflect the head and the tail of the bunches in opposite horizontal directions upstream of the interaction point. At CLIC, the incoming beams must be focused to extremely small spot sizes in order to achieve high charge densities and, in turn, to reach the desired luminosity. As a result, the colliding beams experience very strong electromagnetic fields at the interaction point. The subsequent bending of their trajectories leads to the emission of beamstrahlung photons, which can then turn into $e^{+} e^{-}$pairs.

This paper aims at a conceptual design of the CLIC postcollision beam line, with the following requirements: (i) transport of all charged particles (disrupted beam and $e^{+} e^{-}$pairs) as well as the beamstrahlung photons from the interaction point to their dump, (ii) transport of the non-

*arnaud.ferrari@fysast.uu.se colliding beams to their dump, (iii) controlled beam losses in magnetic elements and collimators along the postcollision line, with a minimal flux of backscattered particles at the interaction point, (iv) implementation of beambased diagnostics to monitor the quality of the $e^{+} e^{-}$ collisions, e.g., for luminosity tuning purposes.

The extraction line once considered for the $20 \mathrm{mrad}$ configuration of the International Linear Collider (ILC), and presently considered for its $14 \mathrm{mrad}$ configuration, consists of a defocusing-focusing-defocusing-focusing quadruplet, just downstream of the interaction point, followed by two vertical magnetic chicanes for energy and polarization measurements, and a long field-free region allowing the beam to naturally grow before it reaches the dump [3]. Such a design is not adapted to CLIC, because of the much larger amount of low-energy charged particles, which would lead to much larger power losses, due to the overfocusing of the low-energy tail by the quadrupoles [4]. At ILC, the beam dump is the primary source of background from backscattered photons and neutrons reaching the interaction point. However, even with a direct line of sight between the dump and the interaction point, the background due to backscattered photons remains much smaller than the beam-beam pair background [5]. As neutrons produced at the dump bounce several times along the post-collision line before reaching the interaction point, the line of sight should not have a significant influence on the associated background. At CLIC, the number of beambeam pairs is larger than at ILC, hence a minimal design in which charged beams and beamstrahlung photons are 
transported together to a common dump, with no magnetic element along their path, could fulfill requirements (i)(iii). On the other hand, a beam line that does not separate the outgoing beams makes the implementation of beambased diagnostics more difficult. Therefore, we propose a design based on the separation of the disrupted beam, the beamstrahlung photons, and the wrong-sign charged particles of the $e^{+} e^{-}$pairs, which is followed by a transport to their respective dump, through dedicated beam lines. The further from the interaction point this separation occurs, the larger the transverse size of the beamstrahlung photon cone and the more difficult the separation, which favors the installation of bending magnets relatively close to the interaction point.

In Sec. II, we review the incoming beam parameters at CLIC, as well as the outgoing beam distributions at the interaction point. We then discuss the extraction and separation of the different components of the outgoing beam, as well as the transport to their respective dump in Sec. III, where we also estimate the losses at various locations along the post-collision line. In Sec. IV, we present a conceptual design study of the vacuum window at the exit of the beam line, $150 \mathrm{~m}$ downstream of the interaction point. In Sec. V, we discuss the implementation of beam-based diagnostics along the post-collision line, with the aim to monitor the quality of the $e^{+} e^{-}$collisions. Finally, a summary is given in Sec. VI.

\section{INCOMING AND OUTGOING BEAMS AT THE INTERACTION POINT}

The incoming beam parameters of the nominal CLIC machine are given in Table I [6]. Note that the transverse rms beam sizes $\sigma_{x, y}^{*}$ take into account radiation effects and nonlinearities in the beam delivery system, and are thus about $30 \%$ larger than the beam sizes derived from linear optics formulas.

TABLE I. Incoming beam parameters at CLIC.

\begin{tabular}{lcc}
\hline \hline Parameter & Symbol & Value \\
\hline Center-of-mass energy & $E_{\mathrm{cm}}$ & $3 \mathrm{TeV}$ \\
Acceleration frequency & $f_{\mathrm{rf}}$ & $12 \mathrm{GHz}$ \\
Acceleration gradient & $g_{\mathrm{ACC}}$ & $100 \mathrm{MV} / \mathrm{m}$ \\
Particles per bunch & $N_{b}$ & $3.72 \times 10^{9}$ \\
Bunches per rf pulse & $n$ & 312 \\
Bunch-bunch spacing & $\Delta t_{b}$ & $0.5 \mathrm{~ns}$ \\
Repetition frequency & $f$ & $50 \mathrm{~Hz}$ \\
Primary beam power & $P_{b}$ & $14 \mathrm{MW}$ \\
Horizontal emittance & $(\beta \gamma) \epsilon_{x}$ & $660 \mathrm{~nm} \mathrm{rad}$ \\
Vertical emittance & $(\beta \gamma) \epsilon_{y}$ & $20 \mathrm{~nm} \mathrm{rad}$ \\
Horizontal rms beam size & $\sigma_{x}^{*}$ & $40 \mathrm{~nm}$ \\
Vertical rms beam size & $\sigma_{y}^{*}$ & $1 \mathrm{~nm}$ \\
Rms bunch length & $\sigma_{z}^{*}$ & $45 \mu \mathrm{m}^{-2} \mathrm{~s}^{-1}$ \\
Peak luminosity & $L$ & $5.9 \times 10^{34} \mathrm{~cm}^{-1}$ \\
Beamstrahlung & $\delta_{B}$ & $29 \%$ \\
\hline \hline
\end{tabular}

The outgoing beam distributions at the interaction point were computed with GUINEA-PIG [7], using Gaussian incoming beams. The angular distributions and the energy spectrum of the disrupted beam are shown in Figs. 1 and 2 respectively, for ideal $e^{+} e^{-}$collisions, i.e., with no beambeam offset. The double-peak shape of the $x^{\prime}$ distribution results from the electric field of the target beam and is characteristic for collisions with flat beams.

Strong beam-beam interactions lead to an increase of the angular divergences and thus a significant emittance growth. The long low-energy tail of the disrupted beam results from beamstrahlung photon emission during the bunch crossing. In average, 2.2 photons are produced per incoming electron or positron, and the energy loss of each incoming beam through this process is $\delta_{B}=29 \%$. The angular distributions of the beamstrahlung photons are shown in Fig. 3. Both the beam-beam interaction and the beamstrahlung photon emission strongly depend on the dimensions and charges of the incoming beams, via the parameter $Y[8]$ :
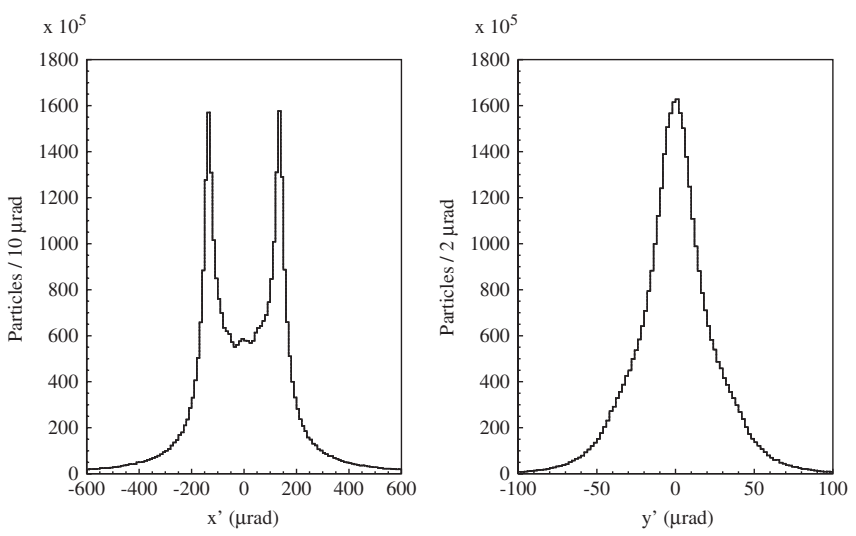

FIG. 1. Horizontal and vertical angular distributions of the CLIC disrupted beam, obtained with GUINEA-PIG using Gaussian incoming beams and normalized to one bunch.

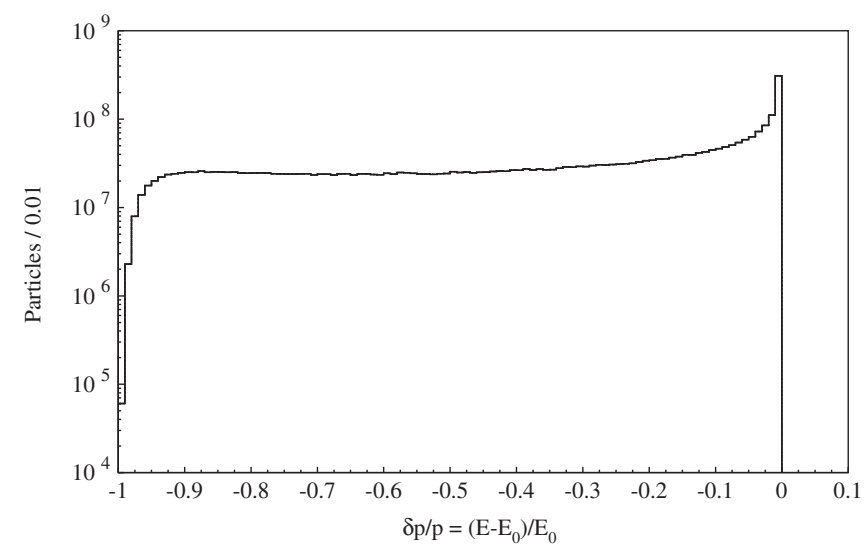

FIG. 2. Relative energy spectrum of the CLIC disrupted beam, obtained with GUINEA-PIG using Gaussian incoming beams and normalized to one bunch. 

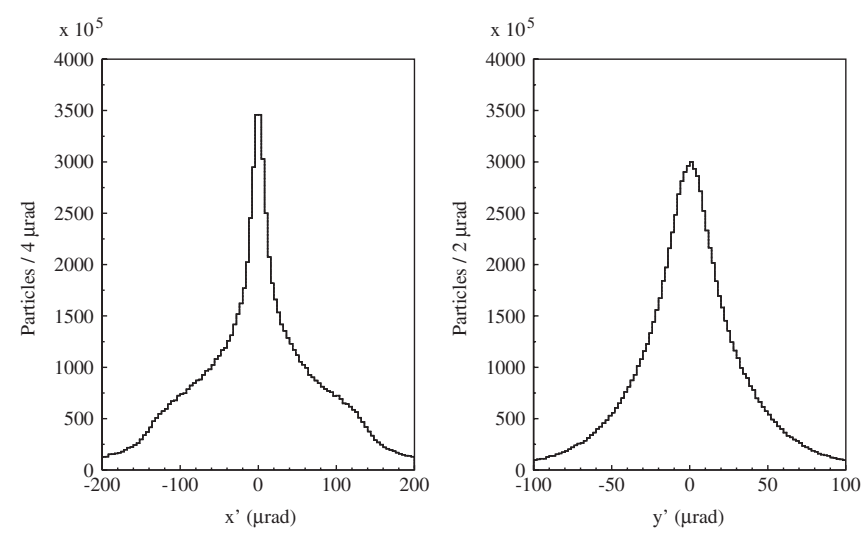

FIG. 3. Horizontal and vertical angular distributions of the beamstrahlung photons, obtained with GUINEA-PIG using Gaussian incoming beams and normalized to one bunch.

$$
\Upsilon=\frac{5}{6} \frac{\gamma r_{e}^{2} N_{b}}{\alpha \sigma_{z}^{*} \sigma_{y}^{*}\left(1+\sigma_{x}^{*} / \sigma_{y}^{*}\right)}
$$

where $\alpha=1 / 137$ and $r_{e}=2.82 \times 10^{-15} \mathrm{~m}$ are the fine structure constant and the classical electron radius. At CLIC, $Y \simeq 5.4$, while it is only 0.046 at ILC.

In a strong electromagnetic field, the beamstrahlung photons can turn into $e^{+} e^{-}$coherent pairs, where the photon interacts with the collective field of the incoming beam. At CLIC, one expects $5 \times 10^{8}$ such $e^{+} e^{-}$pairs per bunch crossing. The presence of a large number of coherently produced $e^{+} e^{-}$pairs in the outgoing beams is a unique feature for CLIC, as compared with other linear colliders, such as ILC. Their transverse distributions and their energy spectrum are shown in Figs. 4 and 5, respectively.

Additional $e^{+} e^{-}$pairs can also be produced via three incoherent processes [9]: $\gamma \gamma \rightarrow e^{+} e^{-}$(Breit-Wheeler), $e \gamma \rightarrow e e^{+} e^{-} \quad$ (Bethe-Heitler), and $e e \rightarrow e e e^{+} e^{-}$ (Landau-Lifshitz). Our GUINEA-PIG simulations suggest that $4.4 \times 10^{5}$ incoherent $e^{+} e^{-}$pairs are produced per
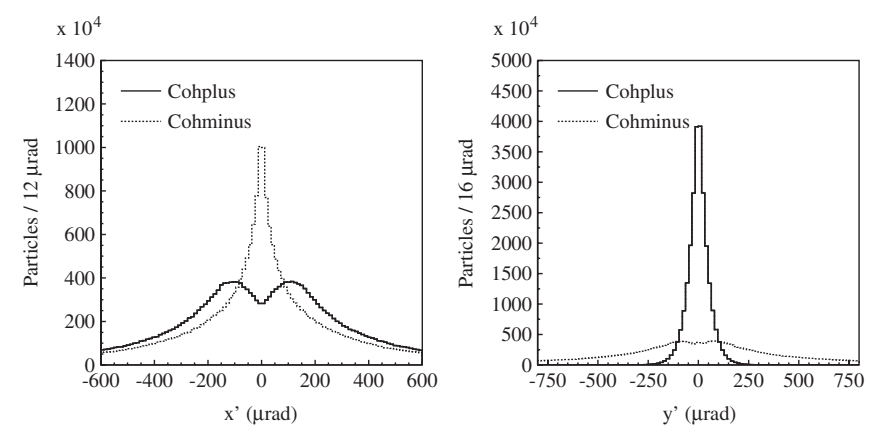

FIG. 4. Horizontal and vertical angular distributions of the particles of the $e^{+} e^{-}$coherent pairs with the same charge as the CLIC disrupted beam (Cohplus) and with the opposite charge (Cohminus), obtained with GUINEA-PIG using Gaussian incoming beams and normalized to one bunch.

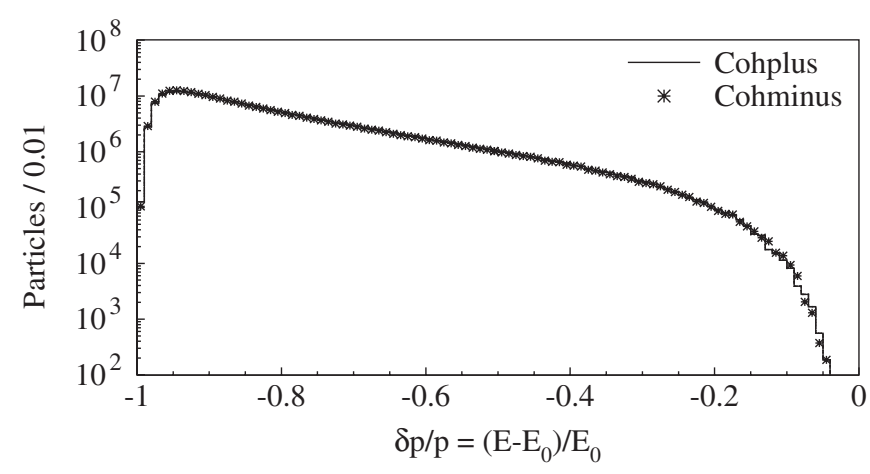

FIG. 5. Relative energy spectrum of the particles of the $e^{+} e^{-}$ coherent pairs with the same charge as the disrupted beam (Cohplus) and with the opposite charge (Cohminus), obtained with GUINEA-PIG using Gaussian incoming beams and normalized to one bunch.

bunch crossing at CLIC. Most of them have a low energy, as well as a large angle with respect to the outgoing beam. Hence, a significant fraction of the incoherent $e^{+} e^{-}$pairs will most likely not be transported in the post-collision line. Instead, they can be used as a signal for luminosity monitoring near the interaction point, as proposed in [10]. The total energy carried away by the incoherent $e^{+} e^{-}$pairs is $5 \mathrm{~mJ}$ per bunch crossing, which corresponds to a total power of $78 \mathrm{~W}$. Hence, in the following, their impact is neglected.

Another source of energy loss for the incoming beams is radiative Bhabha scattering: $e^{+} e^{-} \rightarrow e^{+} e^{-} \gamma$, where the exchange of a virtual photon may induce the emission of a real photon by one incoming electron or positron. At CLIC, one expects $5 \times 10^{5}$ radiative Bhabhas per outgoing bunch. Their energy spectrum is shown in Fig. 6. In contrast with those coming from the incoherent pairs, the

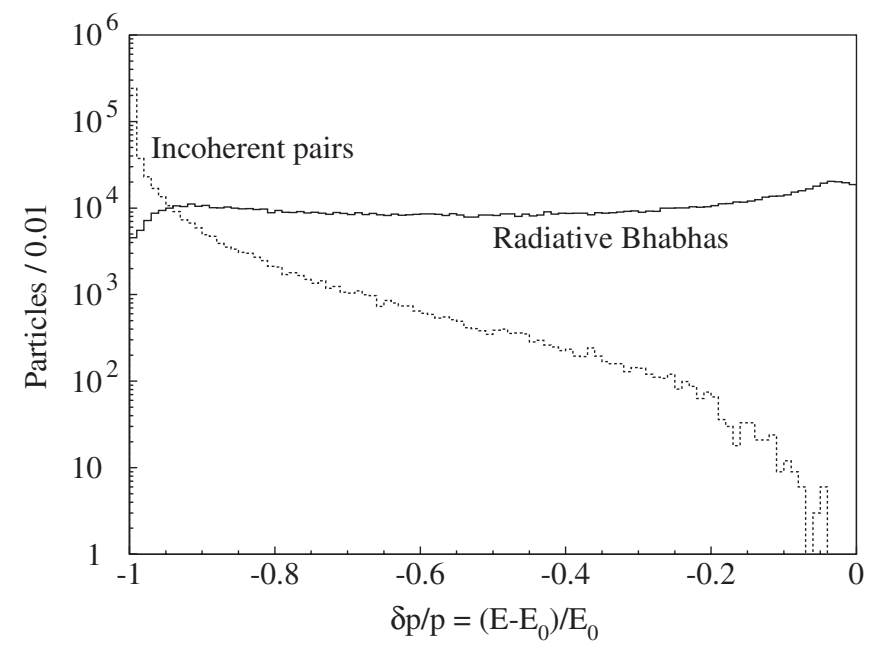

FIG. 6. Relative energy spectrum of the electrons and positrons coming from the radiative Bhabha scattering (full line) and from the incoherent pair production (dashed line). This plot shows the amount of particles per bunch crossing. 
electrons and positrons produced by this process may carry up to $100 \%$ of the primary beam energy. Even if they only carry a tiny fraction of the outgoing beam power (about $1 \mathrm{~kW}$ out of $14 \mathrm{MW}$ ), the low-energy tail of the radiative Bhabhas may lead to small losses along the post-collision line.

\section{CONCEPTUAL DESIGN OF THE CLIC POST- COLLISION LINE}

The design that we propose for the CLIC post-collision line is based on the separation by a magnetic chicane of the disrupted beam, the beamstrahlung photons, and the $e^{+} e^{-}$ coherent pairs, just downstream of the interaction point (see Fig. 7). Obviously, due to the presence of the incoming beam line, the chicane must be vertical. The main constraints used in our study are summarized in Table II (details are given in the following). Our design being conceptual, technical details on the construction of magnets, collimators, and dumps, as well as simulations of backscattered particle fluxes and component activation, are beyond the scope of this paper.

\section{A. Separation of the outgoing beams}

\section{Conceptual design of window-frame magnets}

The first elements of the CLIC post-collision line are four vertically bending sections, spaced by $4 \mathrm{~m}$, each with a field of $0.8 \mathrm{~T}$ and a length of $4 \mathrm{~m}$ (the bending angle provided by each section is $0.64 \mathrm{mrad}$ at $1.5 \mathrm{TeV}$ ). In order to keep the power losses in the magnets as low as possible (in any case, below $10 \mathrm{~W}$ per magnet), while keeping their transverse dimensions at a reasonable level, collimators must be installed between two consecutive bending sections, in order to absorb the low-energy particles far away from the reference beam trajectory. In our design, these collimators are $90 \mathrm{~cm}$ long. With suitable cooling, they can withstand power losses up to $10 \mathrm{~kW}$ [11].

Note that the magnetic field plays a major role in the performance of the post-collision line and needs careful optimization. With fields above $1 \mathrm{~T}$, the vertical extension of the disrupted beam becomes so large that power losses (on the collimators but, even more seriously, inside the magnets) reach unacceptable levels. Weaker fields help protect the beam line elements, but they lead to a worse separation between the beamstrahlung photons and the $1.5 \mathrm{TeV}$ peak of the charged beams, especially with a small beam-beam offset at the interaction point, which enhances the transverse dimensions of the photon cone. In order to efficiently separate the outgoing beams, we require the vertical dispersion to exceed $3 \max \left(\sigma_{\gamma}\right)$ at the intermediate dump.

The magnets used to separate the outgoing beams are (compact) window-frame dipoles. A schematic layout of their cross section is shown in Fig. 8. The distance from the interaction point to the entrance of the first magnet is $L_{\mathrm{IP}}=$ $27.5 \mathrm{~m}$, so that it can be placed outside the detector. In order to keep the power losses as low as possible in the first bending section, it must be divided into two magnets (the first one with a length of $0.5 \mathrm{~m}$ and the second one with a length of $3.5 \mathrm{~m}$ ) and a $50 \mathrm{~cm}$ long collimator must be installed in the $2.5 \mathrm{~m}$ drift space between these two magnets.

In the vertical direction, as the spacing between the beamstrahlung photons and the charged beams increases, so do their vertical sizes, due to their large energy spread. Meanwhile, the horizontal beam size increases linearly with the distance to the interaction point (the most stringent constraint comes from the particles of the coherent pairs with the wrong-sign charge). In order to avoid the (elliptical) vacuum pipe to collapse as a result of the air pressure on its outer side, the thickness of its wall should be

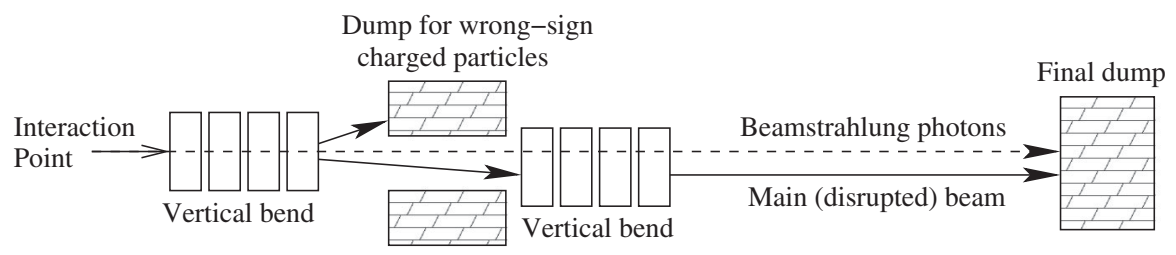

FIG. 7. Schematic layout of the CLIC post-collision line.

TABLE II. Summary of the constraints used for the design of the CLIC post-collision line.

Transport of the $1.5 \mathrm{TeV}$ nominal noncolliding beam

Power losses while transporting the disrupted beam

Separation beamstrahlung photons/charged beams Design of the window-frame and C-type magnets $\sigma_{x} \sigma_{y}$ should exceed $1 \mathrm{~mm}^{2}$ at the main dump. Below $10 \mathrm{~W}$ in the magnets.

Below $10 \mathrm{~kW}$ on the collimators.

A few hundred $\mathrm{kW}$ on the intermediate dump.

The vertical dispersion must exceed $3 \max \left(\sigma_{\gamma}\right)$.

The magnetic field should not exceed $1 \mathrm{~T}$.

Sufficient separation from the incoming beam line. 


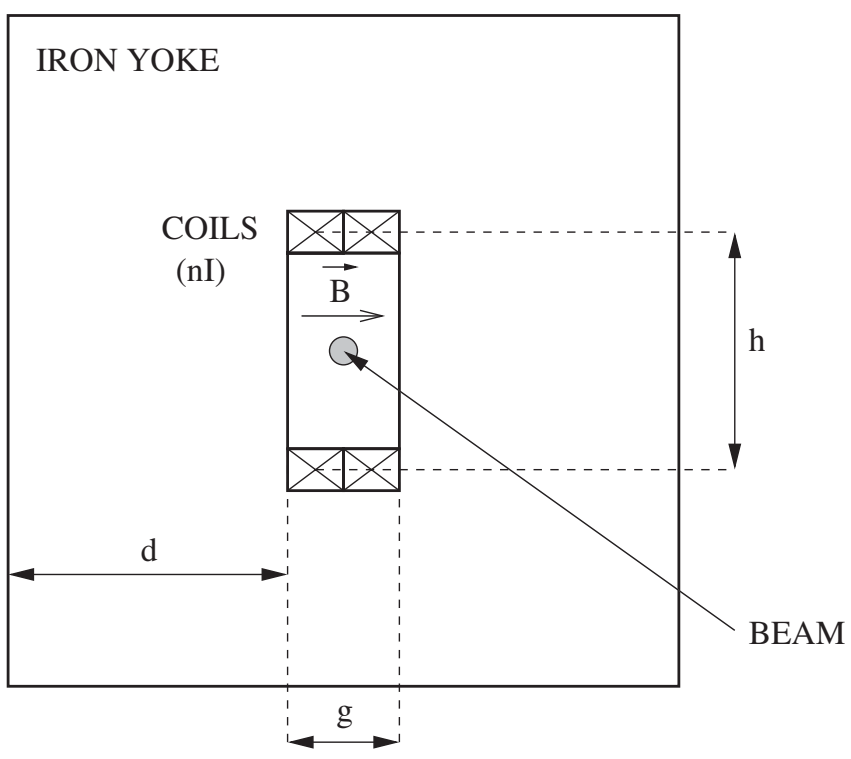

FIG. 8. Cross section of a window-frame magnet, with the relevant parameters to be considered for its design.

larger along the horizontal direction than along the vertical one. In the following, the thickness $T$ of the beam pipe wall is set to $5 \mathrm{~mm}$ along the vertical axis and we assume that the ratio between $T_{x}$ and $T_{y}$ is the same as between the transverse apertures of the pipe. As for the coil, we assume that half of the cross section is used for cooling. With a current density $J=10 \mathrm{~A} / \mathrm{mm}^{2}$ and $X_{\text {coil }}=g$, we find that

$$
Y_{\text {coil }}=\frac{B g}{\mu_{0}} \times \frac{1}{J X_{\text {coil }}}=12.7 \mathrm{~cm} .
$$

Taking into account all constraints, one obtains

$$
\begin{gathered}
h[\mathrm{~cm}]=Y_{\text {pipe }}+Y_{\text {coil }}+2 T_{y}=Y_{\text {pipe }}+13.7 \mathrm{~cm}, \\
g[\mathrm{~cm}]=X_{\text {pipe }}+2 T_{x}=X_{\text {pipe }}+1 \mathrm{~cm} \times \frac{Y_{\text {pipe }}}{X_{\text {pipe }}} .
\end{gathered}
$$

Finally, one must make sure that the iron yoke is large enough in order to allow the magnetic flux to fully return through it. If the maximal field strength in the iron is $B_{\max }=1.7 \mathrm{~T}$, then one must impose

$$
d \geq h \times \frac{B}{2 B_{\max }} .
$$

The geometrical characteristics of each window-frame magnet are summarized in Table III. Horizontally, the spacings between the post-collision and incoming beam lines $(55,76,92$, and $108 \mathrm{~cm}$ at the entrance of the first, second, third, and fourth bending section, respectively) are large enough to allow insertion of all magnets.
TABLE III. Main properties of the window-frame magnets at the beginning of the CLIC post-collision line.

\begin{tabular}{lccccccc}
\hline \hline $\begin{array}{l}\text { Magnet } \\
\text { name }\end{array}$ & $\begin{array}{l}s_{\text {start }} \\
(\mathrm{m})\end{array}$ & $\begin{array}{l}X_{\text {pipe }} \\
(\mathrm{cm})\end{array}$ & $\begin{array}{c}Y_{\text {pipe }} \\
(\mathrm{cm})\end{array}$ & $\begin{array}{c}g \\
(\mathrm{~cm})\end{array}$ & $\begin{array}{c}h \\
(\mathrm{~cm})\end{array}$ & $\begin{array}{c}n I \\
(\mathrm{kA} . t \mathrm{rns})\end{array}$ & $\begin{array}{c}d+g / 2 \\
(\mathrm{~cm})\end{array}$ \\
\hline Mag1a & 27.5 & 20 & 44.0 & 22.2 & 57.7 & 141.3 & 24.7 \\
Mag1b & 30.5 & 20 & 44.0 & 22.2 & 57.7 & 141.3 & 24.7 \\
Mag2 & 38.0 & 27 & 70.2 & 29.6 & 83.9 & 188.4 & 34.6 \\
Mag3 & 46.0 & 34 & 102.0 & 37.0 & 115.7 & 235.5 & 45.7 \\
Mag4 & 54.0 & 41 & 139.4 & 44.4 & 153.1 & 282.6 & 58.2 \\
\hline \hline
\end{tabular}

\section{Physical separation of the outgoing beams}

Downstream of Mag4, at $67 \mathrm{~m}$ from the interaction point, the particles of the coherent pairs with the wrongsign charge are physically separated from the other outgoing beams, as shown in Fig. 9.

In the separation region, the vertical dispersion $D_{y}$ (and therefore the distance between the center of the beamstrahlung photon cone and the $1.5 \mathrm{TeV}$ reference wrong-sign charged particle of the coherent pairs) is $5.9 \mathrm{~cm}$. GUINEAPIG simulations with small beam-beam offsets suggest that the largest vertical angular divergence of the beamstrahlung photons is $0.2 \mathrm{mrad}$, which leads to a maximal vertical cone size of $1.3 \mathrm{~cm}$ (rms) in the separation region, i.e., 4.5 times smaller than $D_{y}$. Along the $9 \mathrm{~m}$ long drift between the exit of Mag4 and the separation region, $X_{\text {pipe }}$ and $Y_{\text {pipe }}$ increase from 41 to $54 \mathrm{~cm}$ and from 1.4 to $2.5 \mathrm{~m}$, respectively, in order to avoid power losses.

The transverse beam profiles in the separation region, $67 \mathrm{~m}$ after the interaction point, are shown in Fig. 10.

The stars show the inner wall of the common vacuum chamber, prior to the separation $\left(X_{\text {pipe }}=54 \mathrm{~cm}\right.$ and $\left.Y_{\text {pipe }}=250 \mathrm{~cm}\right)$. For the beamstrahlung photons, the disrupted beam and the same-sign charged particles of the coherent pairs, the vacuum pipe after the physical separation consists of two joined half ellipses. The upper one has its origin at the center of the beamstrahlung photon cone, and its two semiaxis are $X_{\text {up }}=27 \mathrm{~cm}$ and $Y_{\text {up }}=5 \mathrm{~cm}$. The lower one is centered on the path of the $1.5 \mathrm{TeV}$ reference particle of the main beam, with $X_{\text {down }}=27 \mathrm{~cm}$ and $Y_{\text {down }}=119 \mathrm{~cm}$. The elliptical exit window for the

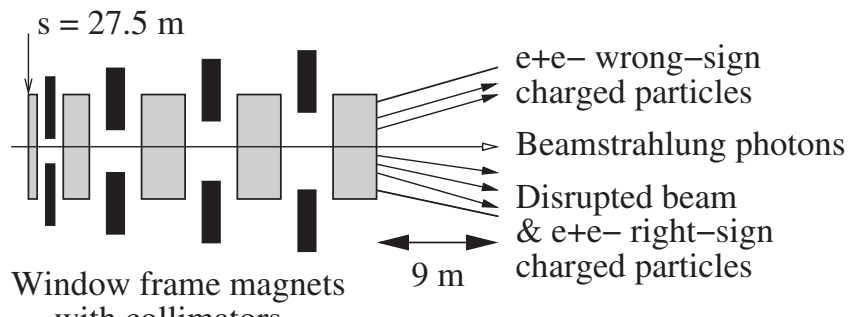
with collimators

FIG. 9. Schematic layout of the first $67 \mathrm{~m}$ of the CLIC postcollision line, where the separation of the various components of the outgoing beam is performed. 


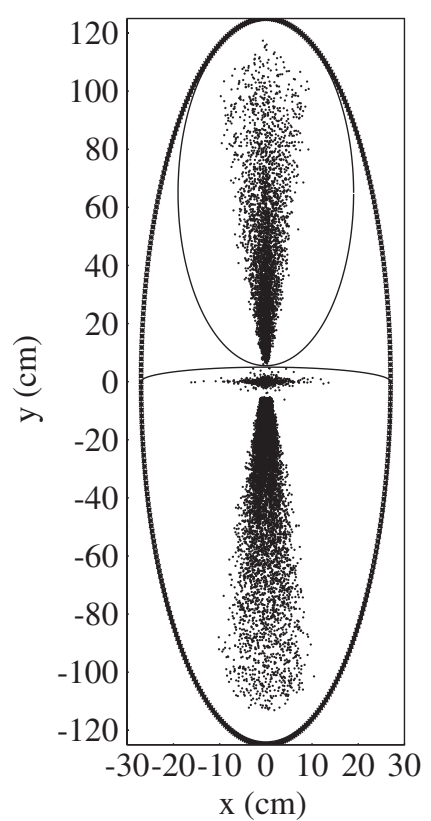

FIG. 10. Transverse beam profiles obtained in the separation region, $67 \mathrm{~m}$ downstream of the interaction point.

wrong-sign charged particles of the coherent pairs is also shown (its horizontal and vertical semiaxis are 19 and $59 \mathrm{~cm}$, respectively).

In order to recover information on the coherent pairs, only the particles with the wrong-sign charge can be used, since the other ones cannot be distinguished from the lowenergy tail of the disrupted beam. Therefore, it is crucial to collect as many particles as possible, in order to derive the full energy spectrum of the coherent pairs from the vertical beam profile of the particles with the wrong-sign charge.

The left-hand side plot of Fig. 11 shows the correlation between the vertical position and the energy for the particles of the coherent pairs with the wrong-sign charge after their separation from the other components of the outgoing
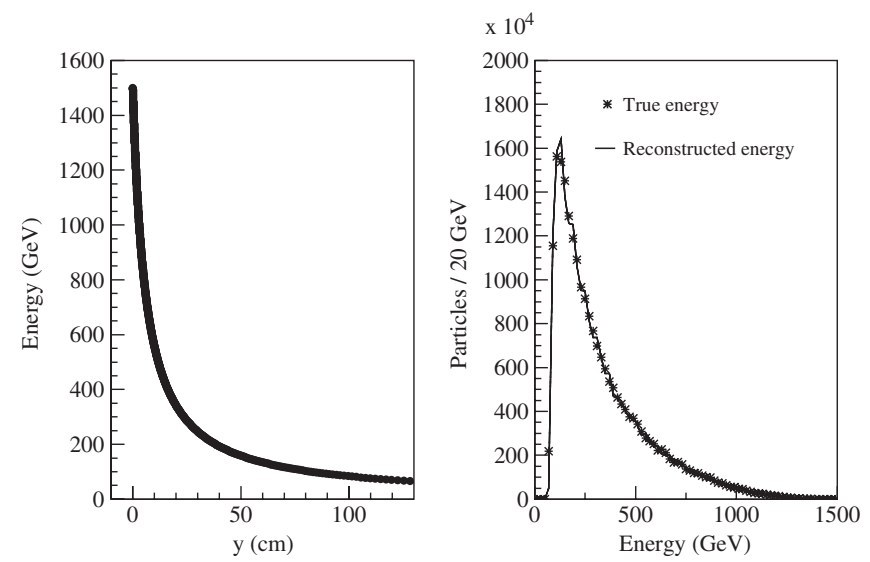

FIG. 11. Reconstructing the energy spectrum of the coherent pairs from the vertical beam profile of the wrong-sign charged particles just after the separation region. beam, $67 \mathrm{~m}$ after the interaction point (these particles were generated with $x=y=0$ and $x^{\prime}=y^{\prime}=0$ ). On the righthand side plot, the energy spectrum derived from the vertical beam profile is compared with the true one. An excellent agreement is obtained, suggesting that the energy distribution of the coherent pairs can be efficiently retrieved with this method (the angle $y_{\mathrm{IP}}^{\prime}$ of any given particle at the interaction point has practically no impact on its position downstream of the window-frame magnets).

In addition to the energy spectrum, one should also measure the number of $e^{+} e^{-}$coherent pairs, through the total power carried by the wrong-sign charged particles, since this parameter strongly depends on small beam-beam offsets at the interaction point and is therefore very useful to control the quality of the collisions.

\section{B. Transport of the main disrupted beam and the beamstrahlung photons to their dump}

Synchrotron radiation is emitted by all charged particles inside the extraction magnets, and it is mostly found between the beamstrahlung photon cone and the high-energy edge of the main charged beam (the intensity and the power density of the synchrotron radiation is much lower for the coherent pairs). Any piece of material used in order to physically separate the main beam from the beamstrahlung photons may thus be severely damaged by synchrotron radiation. Hence, a common vacuum pipe is used for the beamstrahlung photons and the disrupted beam. In addition, one needs the exit window between the accelerator vacuum and the dump of the main beam to be far away from the interaction point, so that the transverse sizes of the noncolliding beam can grow up to a few $\mathrm{mm}$. The deflection angle provided by the window-frame magnets must be followed by a bend in the opposite direction, in order to rapidly have $D_{y}^{\prime}=0$ at the exit of the vertical chicane (and at the dump). For this purpose, four C-type dipole magnets are used. Each of them has a length of $4 \mathrm{~m}$ and a field strength of (about) $0.8 \mathrm{~T}$. Two consecutive C-type magnets are spaced by $3 \mathrm{~m}$. They are placed after the dump of the wrong-sign charged particles of the coherent pairs in order to avoid encumbrance problems, see Fig. 12. A schematic layout of the C-type magnets is given in Fig. 13.

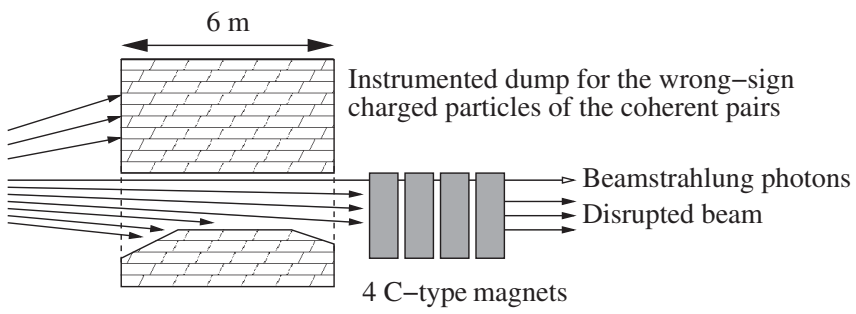

FIG. 12. Schematic layout of the transport line between the separation region and the C-type magnets, which passes through the instrumented dump of the wrong-sign charged particles of the coherent pairs. 


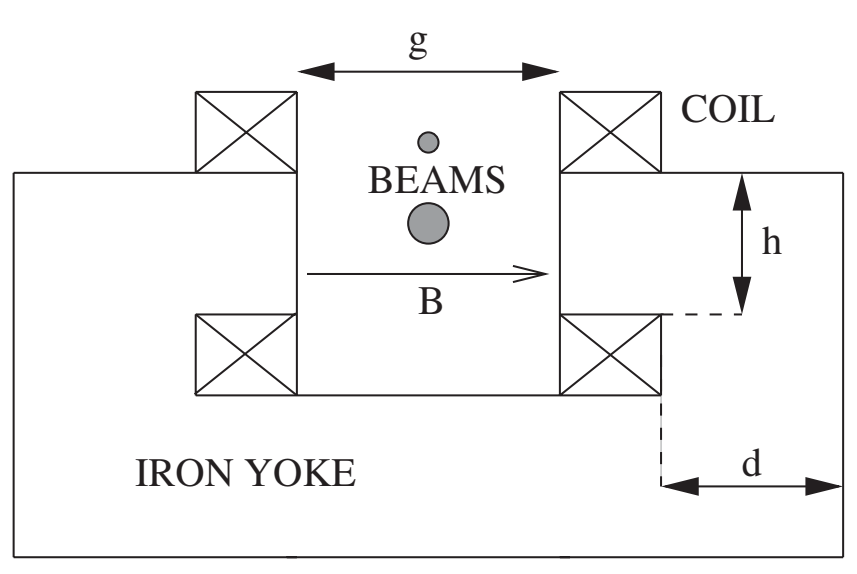

FIG. 13. Cross section of a C-type magnet, with the relevant parameters to be considered for its design. The disrupted beam passes between the poles, while beamstrahlung photons travel between the upper coils.

Just after the separation region, the disrupted beam and the same-sign charged particles of the coherent pairs, as well as the beamstrahlung photons, pass through the $6 \mathrm{~m}$ long dump, before reaching the first C-type magnet, installed $2 \mathrm{~m}$ downstream. The transverse dimensions of the vacuum pipe that goes through the dump, and in turn the losses occurring there, will be discussed later, as they depend on the design of the C-type magnets.

In the following, we use $g=45 \mathrm{~cm}$. In that case, an excitation current of $288 \mathrm{kA}$.turns is required in order to produce a field of $0.8 \mathrm{~T}$ in the gap. This leads to a cross section of $576 \mathrm{~cm}^{2}$ for the coils, if we assume that half of it is used for cooling and that the current density is $J=10 \mathrm{~A} / \mathrm{mm}^{2}$. The horizontal dimension of the C-type magnet is $g+2\left(d+X_{\text {coil }}\right)$, where the distance $d$ must be large enough to allow the magnetic flux $\Phi$ to fully return through the iron yoke. It is rather trivial to analytically calculate $\Phi$ in the lateral parts of the magnet, where the iron yoke is thinnest. On the other hand, since the field is not fully contained inside the gap, it is more complicated to estimate $\Phi$ there. The leakage of the flux depends on several parameters, such as the distance between the poles, their shape, the position of the coils, the grade of saturation, etc. There is no simple analytic formula taking into consideration all these aspects. Instead, we consider an ideal configuration, in which the coils are close to the iron, the magnet is not saturated, the flux is entering and leaving the yoke through the (parallel) pole faces only and the distance between the poles is small compared to their width. In that case, about $95 \%$ of the flux is contained in $B \times \ell \times(h+0.5 g)$, where $\ell$ is the length of the C-type magnet. If the maximal field strength inside the iron yoke is $B_{\max }$ (chosen to be $1.7 \mathrm{~T}$ as in the case of the window-frame magnets), then the distance $d$ is roughly constrained by

$$
d \geq \frac{B}{B_{\max }}(h+0.5 g) .
$$

TABLE IV. Main properties of the four C-type magnets.

\begin{tabular}{lc}
\hline \hline Parameter & Value \\
\hline$g$ & $45 \mathrm{~cm}$ \\
$h$ & $75 \mathrm{~cm}$ \\
$n I$ & $288 \mathrm{kA.turns}$ \\
$X_{\text {coil }}$ & $20 \mathrm{~cm}$ \\
$Y_{\text {coil }}$ & $28.8 \mathrm{~cm}$ \\
$d+X_{\text {coil }}+g / 2$ & $88.5 \mathrm{~cm}$ \\
\hline \hline
\end{tabular}

With $h=75 \mathrm{~cm}$, the smallest value of $d$ is $46 \mathrm{~cm}$. If the horizontal and vertical sizes of the excitation coils are set to, respectively, 20 and $28.8 \mathrm{~cm}$, the minimum horizontal half size of the four C-type magnets is $88.5 \mathrm{~cm}$, while the spacing between the post-collision line and the incoming beam line is $1.5 \mathrm{~m}$ at the entrance of the first C-type magnet.

The main geometrical properties of the four C-type magnets are summarized in Table IV.

If the magnetic field is exactly $0.8 \mathrm{~T}$ in all magnets, then the vertical dispersion at the exit of the post-collision chicane is $11.1 \mathrm{~cm}$ for the $1.5 \mathrm{TeV}$ reference particle of the disrupted beam, with $D_{y}^{\prime}=0$ (see Fig. 14).

However, due to synchrotron radiation in the magnets of the post-collision line, the high-energy peak of the disrupted beam has $\delta p / p=-0.038$ at the exit of the last Ctype magnet and is thus slightly displaced. Also, it leaves the vertical chicane with a small positive angle. One way to compensate for this effect is to slightly reduce the field strength in the C-type magnets, down to $0.784 \mathrm{~T}$. In that case, the high-energy peak, now at $1.44 \mathrm{TeV}$, is parallel to the beamstrahlung photons after the magnetic chicane. The $y$ and $y^{\prime}$ distributions of the disrupted beam after the

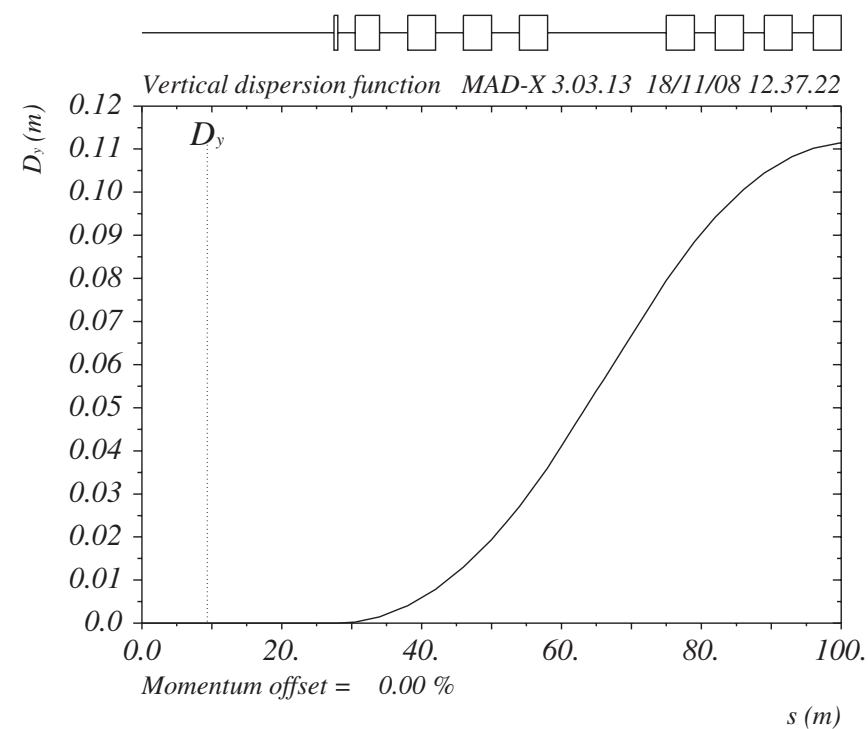

FIG. 14. Dispersion function along the first $100 \mathrm{~m}$ of the CLIC post-collision line, obtained with MAD-X [12]. 

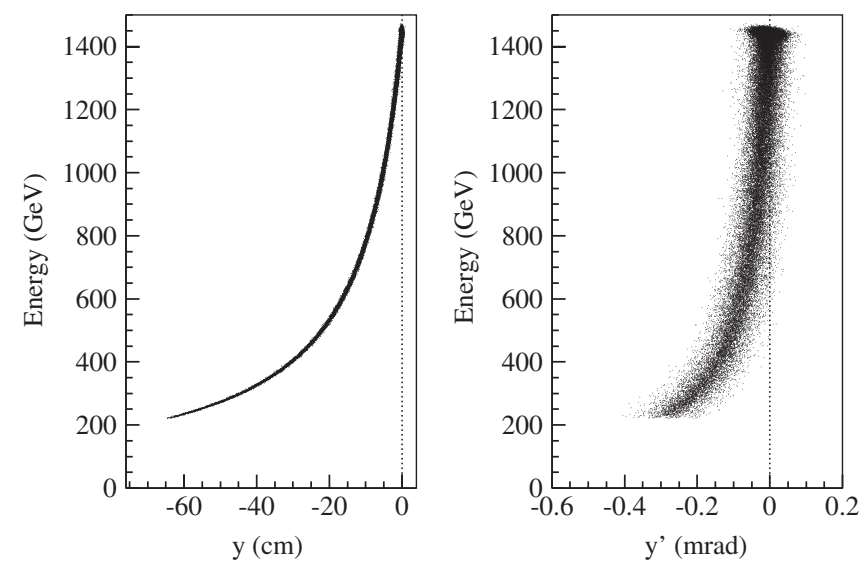

FIG. 15. $y$ and $y^{\prime}$ distributions as a function of the energy for the disrupted beam, after the post-collision chicane.

last C-type magnet (100 $\mathrm{m}$ downstream of the interaction point) are shown in Fig. 15 as a function of the energy. The presence of nonlinear dispersion is clearly visible.

A schematic drawing of the vacuum pipe used for the transport of the disrupted beam and the beamstrahlung photons through the dump and the four C-type magnets is shown in Fig. 16. The half ellipse which defines the lower half aperture of the vacuum pipe is centered on the 1.5 TeV reference particle. The other half ellipse, which defines the upper half aperture of the vacuum pipe, is centered on the beamstrahlung photon cone. Both half ellipses have the same horizontal aperture. The distance between their centers is equal to the vertical dispersion $D_{y}$. The overall shape of the vacuum pipe remains the same from the separation region to the exit of the fourth C-type magnet. However, one must take into account the increasing vertical dispersion. Also, a fraction of the charged particles must be absorbed in the dump in order to protect the four C-type magnets downstream. Hence, the distance between the two half ellipses, as well as their semiaxis, must change along the path of the disrupted beam and the beamstrahlung photons, see Fig. 17.

This design allows one to protect the C-type magnets, as all particles with $\delta p / p<-0.84$ are absorbed in the dump. Along the horizontal direction, the narrow section inside the dump allows one to absorb particles with $\left|x^{\prime}\right|>$ $1.3 \mathrm{mrad}$ before they pass through the C-type magnets.

After the fourth C-type magnet, the beamstrahlung photons and the disrupted beam drift freely towards their dump. The distance between the interaction point and the main dump is constrained by the transverse sizes of the noncolliding beam, as they directly determine the maximal temperature increase and the thermal stress for the exit window at the end of the post-collision line. If the weak focusing of the magnets is neglected, then the transverse sizes of the noncolliding beam at a distance $s$ from the interaction point are given by

$$
\sigma_{x}(s) \simeq \sigma_{x}^{*} \times \frac{s}{\beta_{x}^{*}}
$$

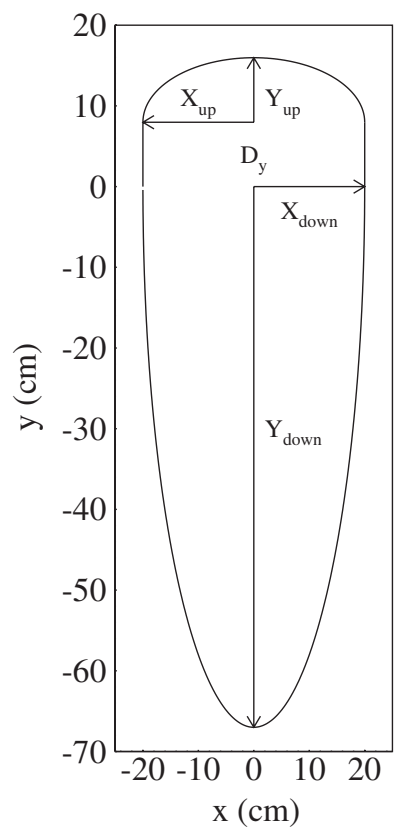

FIG. 16. Schematic drawing of the vacuum chamber for the disrupted beam and the beamstrahlung photons (here $75 \mathrm{~m}$ after the interaction point): two half ellipses with the same horizontal semiaxis but different vertical semiaxis $\left(Y_{\text {down }}\right.$ and $Y_{\text {up }}$ ) are separated by a distance equal to $D_{y}$.

$$
\sigma_{y}(s) \simeq \sqrt{\left(\sigma_{y}^{*} \times \frac{s}{\beta_{y}^{*}}\right)^{2}+\left(D_{y}(s) \frac{\delta_{p}}{p}\right)^{2}},
$$

with $\beta_{x}^{*}=4 \mathrm{~mm}$ and $\beta_{y}^{*}=0.09 \mathrm{~mm}$. In our design, the exit window is placed $150 \mathrm{~m}$ away from the interaction point, where the vertical dispersion is $11.1 \mathrm{~cm}$. Assuming an energy spread of $1 \%$ for the noncolliding beams, $\sigma_{x} \sigma_{y}$ is $3 \mathrm{~mm}^{2}$ at the end of the post-collision line.
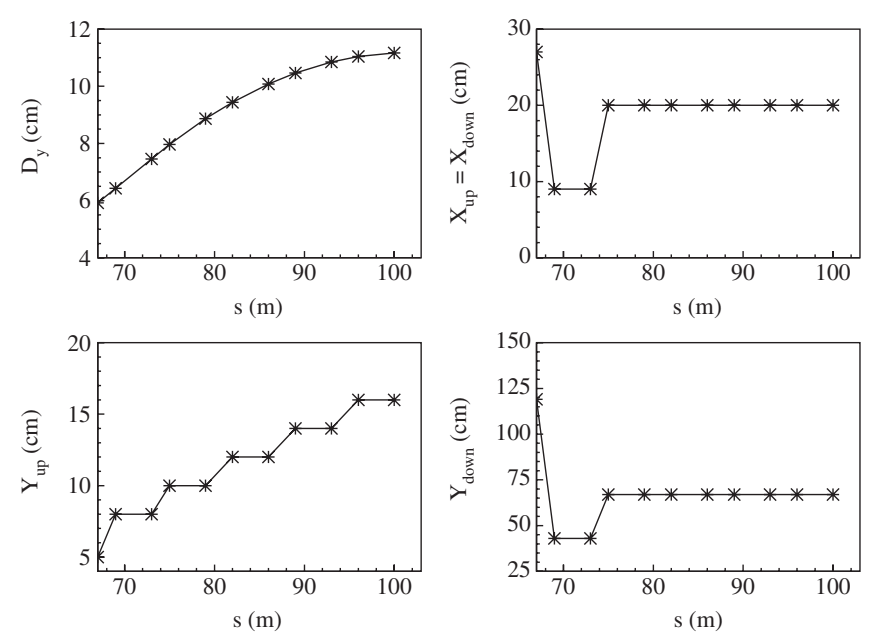

FIG. 17. Variations of the dimensions of the vacuum pipe for the disrupted beam and the beamstrahlung photons, from the separation region to the fourth C-type magnet exit. 


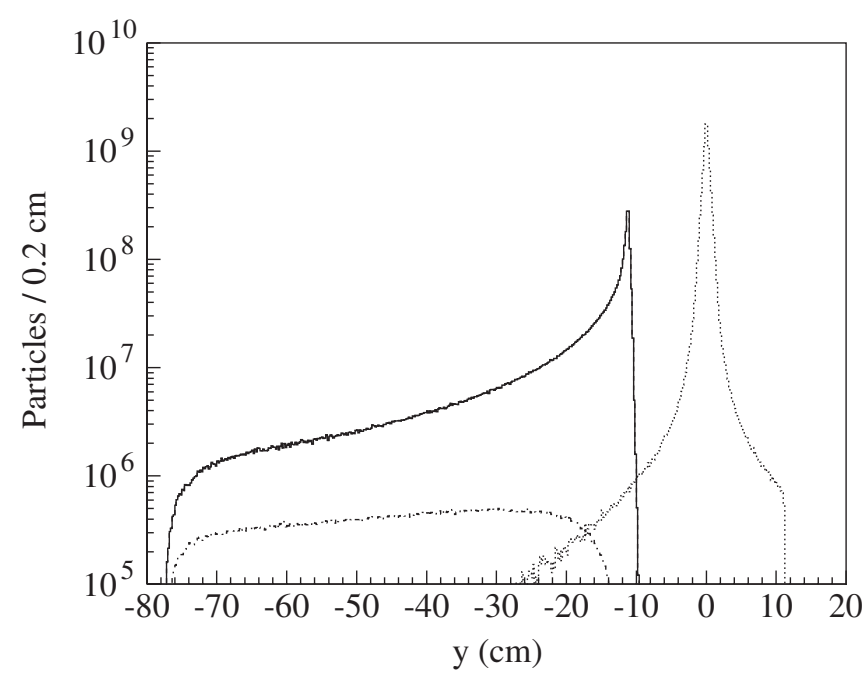

FIG. 18. Vertical profiles for the charged beam (full line), including the particles of the coherent pairs with the right-sign charge (dashed line), and for the beamstrahlung photons (dotted line), as obtained at the end of the post-collision line, $150 \mathrm{~m}$ downstream of the interaction point.

Along the $50 \mathrm{~m}$ long drift from the last C-type magnet to the exit window, the vacuum pipe gradually gets a racetrack shape. At the end of the CLIC post-collision line, the length of the straight line is $54 \mathrm{~cm}$, while the radius of both the upper and lower circles is $20 \mathrm{~cm}$. The upper half circle has its center $4 \mathrm{~cm}$ below the axis of the beamstrahlung photon cone.

The vertical beam profiles obtained at the end of the post-collision line, $150 \mathrm{~m}$ downstream of the interaction point, are shown in Fig. 18.

Should the deposited energy density be reduced in the water-based dump, there is enough space for installing sweeping magnets downstream of the vertical chicane. Should the vertical size of the exit and dump windows be decreased, as compared to the present design, additional collimators or C-type magnets can be implemented as well, in order to, respectively, absorb or bend upwards particles in the low-energy tails. Indeed, the design of the last section of the post-collision line allows for flexibility and can easily be modified without significantly affecting the overall performance of the beam line.

\section{Beam losses along the CLIC post-collision line}

In order to make a first estimate of the performance of the CLIC post-collision line in terms of beam losses, tracking simulations were performed with DIMAD [13]. It computes particle trajectories using the second order matrix formalism [14], at all orders for energy deviations. By default, every element defined in DIMAD is placed on a reference trajectory determined by the nominal $1.5 \mathrm{TeV}$ beam. However, until the separation region, all magnets and collimators are centered on the (straight) path of the beamstrahlung photons. As a result, one must introduce small vertical misalignments when tracking the charged beams. In DIMAD, as soon as an electron, positron, or photon hits a collimator, it is removed from the list of tracked particles. Having introduced (hard-edged) aperture limitations along the post-collision line, the power losses (in Watts) are estimated using

$$
P_{\text {loss }}=1.602 \times 10^{-10} \frac{N_{b} n f}{N_{\text {tracks }}} \sum_{i=1}^{N_{\text {loss }}} E_{i},
$$

where $E_{i}$ is the energy of the lost particle $i$ (in $\mathrm{GeV}$ ), $N_{\text {tracks }}$ and $N_{\text {lost }}$ are, respectively, the number of tracked and lost particles.

Since the total power loss of the radiative Bhabhas in the CLIC post-collision line is $22 \mathrm{~W}$ only, these will not be considered in the following discussion.

For the disrupted beam and the coherent pairs, losses occur mostly in the collimators and the first dump, and they are clearly related to the energy deviation. The vertical aperture limitations seen by the disrupted beam and the same-sign charged particles of the coherent pairs, from the entrance of the first window-frame magnet to the exit of the fourth C-type magnet, are shown in Fig. 19. The distribution of the losses for the charged beams is shown in Fig. 20.

The power losses of the wrong-sign charged particles of the coherent pairs are $1.6 \mathrm{~W}$ and $2.3 \mathrm{~W}$ in Mag1a and Mag1b, respectively. The disrupted beam and the samesign charged particles of the coherent pairs deposit power ( $3 \mathrm{~W}$ ) in Mag1b only. The beam transport through Mag2, Mag3, and Mag4 is loss free. All charged particles with $\delta p / p>-0.95$ reach the separation region. Downstream of the first dump, all particles with $\delta p / p>-0.84$ are transported through the C-type magnets without loss and reach the final dump. A summary of the apertures chosen for the collimators and the dump, as well as the corresponding power losses, are given in Table V. Collimator 0 is the $50 \mathrm{~cm}$ long collimator placed between the first two magnets, while collimator 12 , collimator 23 , and collima-

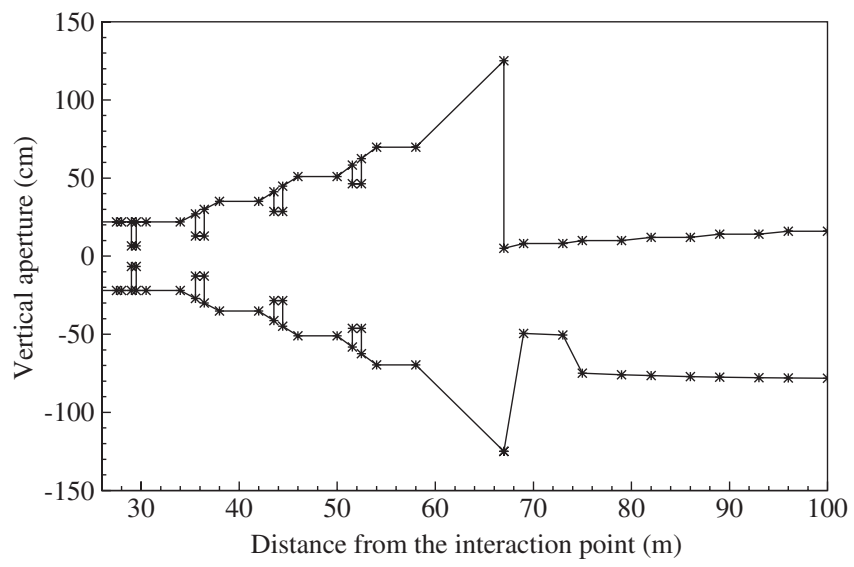

FIG. 19. Vertical apertures seen by the disrupted beam and the same-sign charged particles of the coherent pairs. 


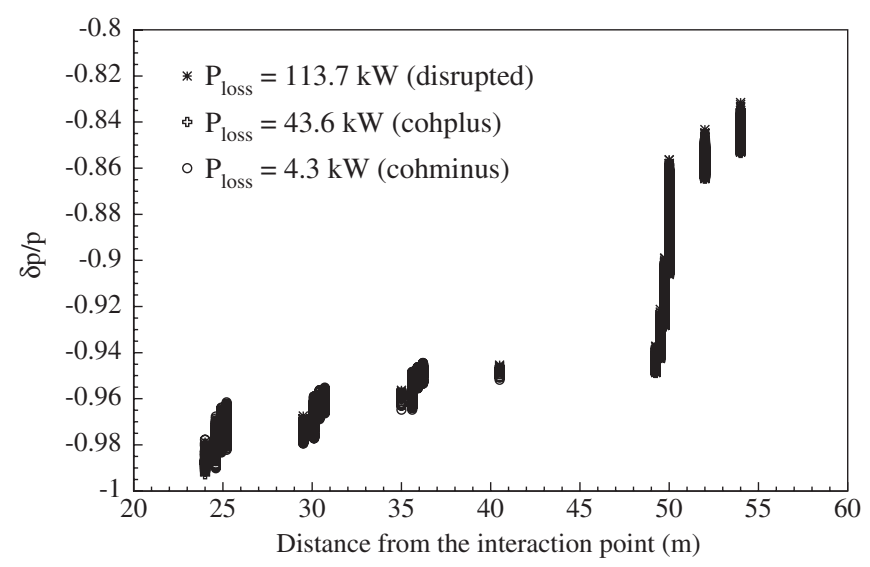

FIG. 20. Relative energy spread of the lost particles as a function of the position of loss in the CLIC post-collision line, obtained when tracking the charged beams. The wrong-sign charged particles absorbed in their dump are not shown.

tor 34 are the three $90 \mathrm{~cm}$ long collimators placed between the $4 \mathrm{~m}$ long bending sections.

Since beamstrahlung photons follow straight trajectories along the post-collision line, their power losses only depend on the angular divergences at the interaction point. These losses (about $100 \mathrm{~W}$ ) occur mostly in the intermediate dump upstream of the C-type magnets.

If the disruption process is enhanced by offsets between the incoming beams, then the beamstrahlung parameter and the amount of $e^{+} e^{-}$coherent pairs may increase, which results in larger losses, especially for the charged beams. However, these mostly occur in the collimators and the intermediate dump.

All tracking simulations were performed with DIMAD and successfully cross-checked with BDSIM [15]. This program is based on GEANT4 [16] and provides a toolkit in order to fully simulate the interactions between the lost particles and the surrounding matter. Indeed, charged and neutral lost particles shower electromagnetically or hadronically in the collimators and the dumps, thereby producing a flux of backscattered background particles, which can be a concern if they irradiate the detector, causing radiation issues or providing a background to the signals from physics. At this point, we briefly consider these backgrounds and comment on prospects for future, more detailed analysis. The background species at the interaction point include photons and neutrons, while the dominant locations for the losses coming from charged particles are the collimators sandwiched between the first vertical bends, the intermediate dump, and the main dump. As for the beamstrahlung photons, they may produce a significant background flux towards the interaction point from the final dump only.

As neutrons may bounce several times along the CLIC post-collision line, the corresponding background at the interaction point can arise from all loss locations. It is however likely to be dominated by the water-based main dump, hence irrespectively of the chosen geometry for the post-collision line.

Let us now consider the electromagnetic background, i.e., the backscattered photons reaching the interaction point. Their energy spectrum is dominated by the range corresponding to Compton photons, which are produced quasi-isotropically in angle. The background studies performed in [17] for the $2 \mathrm{mrad}$ configuration of the ILC focused on the beam losses in the first collimator of the extraction line, positioned a few tens of meters from the interaction point and taking a load at the $\mathrm{kW}$ level. The calculations showed that Compton photons produced on the collimator have a very low probability of reflection. The resulting flux at the interaction point is dominated by photons produced with a direct line of sight. Moreover, these photons lead to a negligible number of hits in the inner layers of the detector, as compared to the hits coming from the incoherent pairs. The studies performed in [17] are very similar to the electromagnetic background calculations for the geometry of this paper, with the difference of a larger number of vertex detector hits coming from the incoherent pairs at CLIC, due to stronger beam-beam interaction. Hence, we expect the photon flux coming from the losses in collimator 0 (the only collimator with a direct line of sight to the interaction point) to remain at an acceptable level. Note also that, in terms of background level due to backscattered photons, losses at the $\mathrm{kW}$ level at about $30 \mathrm{~m}$ from the interaction point are much safer than a single $14 \mathrm{MW}$ loss with a direct line of sight, even $150 \mathrm{~m}$

TABLE V. Power losses for the disrupted beam and for the coherent pairs in the collimators and the intermediate dump.

\begin{tabular}{lcccc}
\hline \hline & Half-aperture & \multicolumn{3}{c}{ Beam losses in kW } \\
Element name & Limitation & Main beam & Cohplus & Cohminus \\
\hline Collimator 0 & $Y_{c}=6.6 \mathrm{~cm}$ & 0 & 0 & 0.98 \\
Collimator 12 & $Y_{c}=12.8 \mathrm{~cm}$ & 0.47 & 0.47 & 3.05 \\
Collimator 23 & $Y_{c}=28.5 \mathrm{~cm}$ & 2.23 & 1.78 & 0.66 \\
Collimator 34 & $Y_{c}=46.3 \mathrm{~cm}$ & 4.21 & 2.72 & 1.89 \\
Dump 1 & $X_{\text {down }}=9 \mathrm{~cm} ; Y_{\text {down }}=43 \mathrm{~cm}$ & 96.2 & 35.2 & 170.1 \\
\hline \hline
\end{tabular}


away, which would be the case if the outgoing beams were not separated.

In the CLIC post-collision line, the second, third, and fourth collimators are screened by the other collimators placed upstream, hence they do not have a direct line of sight to the interaction point. Therefore, the backscattered photons produced by charged beam losses in collimator 12 , collimator 23, and collimator 34 are not likely to result in any hit in the inner detector. From the intermediate dump, there is no line of sight to the interaction point for the main beam and the same-sign charged particles of the coherent pairs. This is however not completely true for the wrongsign charged particles, at least below a certain vertical position on the face of the intermediate dump, i.e., above a certain energy. A third possible source of backscattered photons with a direct line of sight to the interaction point is the beamstrahlung photon spot at the final dump. On the other hand, the spot of the charged beam on the face of the final dump is fully screened by the intermediate dump. Note that, in order to further reduce the electromagnetic background at the interaction point, an additional mask can be installed upstream of the first bending magnet, in the zero-dispersion region. Its aperture must be large enough to let all outgoing beams pass through without any power loss, but small enough to minimize the solid angle seen from the interaction point towards the post-collision line, and thereby the flux of backscattered photons.

The CLIC post-collision line is being modeled with BDSIM, as a preparation for future studies aimed at computing all background rates at the interaction point, as well as their impact on the detector.

\section{DESIGN OF THE VACUUM WINDOW AT THE EXIT OF THE POST-COLLISION LINE}

The vacuum window at the end of the post-collision line must be carefully designed, since it has to withstand a 14 MW beam power, not only when the $e^{+} e^{-}$collisions occur (in which case the charged beams are widened at the interaction point), but also with noncolliding beams which, due to their very low emittances, are much smaller on the window, yielding a very large local energy density.

When designing the exit window, one must make sure that its thickness $d$ remains significantly smaller than one radiation length $X_{0}$. In that case, mostly ionization losses occur in the window. In addition, the exit window must withstand the pressure difference between its two faces. Therefore, the use of a thick window, with a low- $Z$ material, is preferable.

At the Large Hadron Collider, a large diameter carboncarbon composite dump window was designed [18] and the SIGRABOND 1501G grade material from SGL was chosen. Its radiation length is $29 \mathrm{~cm}$, allowing a relatively thick $(15 \mathrm{~mm})$ window and thereby reducing the mechanical stress due to the pressure difference. Also, such a material has both a low elastic modulus $E$ and a low thermal expansion coefficient $\alpha$, which yields a small thermal stress. However, SIGRABOND 1501G is quite porous, hence a thin $(0.2 \mathrm{~mm})$ leak-tight layer is needed to hold vacuum. It can be installed on the high pressure side of the window, since the outgassing rate of the carboncarbon composite is low. If the thin foil is fully supported by the thick window, then the main load comes from the thermal stress and one should choose a material which quickly transports away the heat resulting from the beam impact, for example, aluminum.

The passage of one bunch train, with $n N_{b}$ particles, through the exit window leads to an instantaneous temperature rise $\Delta T$ at the center of the beam spot. With a Gaussian beam and no temperature dependence of the heat capacity $C$, the instantaneous heating is [19]

$$
\Delta T=\left(\frac{d E}{\rho d z}\right) \times \frac{n N_{b}}{2 \pi C \sigma_{\text {beam }}^{2}} .
$$

If $\alpha$ is the thermal expansion coefficient and $E$ is the elastic modulus, the (cyclic) thermal stress due to the (repetitive) temperature increase is

$$
\sigma_{c}=\alpha E \Delta T
$$

The largest temperature rise is caused by a $1.5 \mathrm{TeV}$ noncolliding beam, for which $\sigma_{\text {beam }}^{2}$ is $3 \mathrm{~mm}^{2}$ (assuming an energy spread of $1 \%$ ). The energy deposition along the exit window was computed using FLUKA [20,21], see Fig. 21.

In the carbon-carbon composite $\left(\rho=1.5 \mathrm{~g} / \mathrm{cm}^{3}\right)$, the energy deposition slowly increases with the amount of material seen by the incident beam (from 1.6 to $1.8 \mathrm{MeV} / \mathrm{g} \mathrm{cm}^{-2}$ ), because some particle multiplication occurs, although a full electromagnetic shower has not developed yet. In the aluminum foil $\left(\rho=2.7 \mathrm{~g} / \mathrm{cm}^{3}\right)$, the deposited energy is $1.7 \mathrm{MeV} / \mathrm{g} \mathrm{cm}^{-2}$. The properties of the materials, the instantaneous temperature rise, and the cyclic thermal stress are summarized in Table VI.

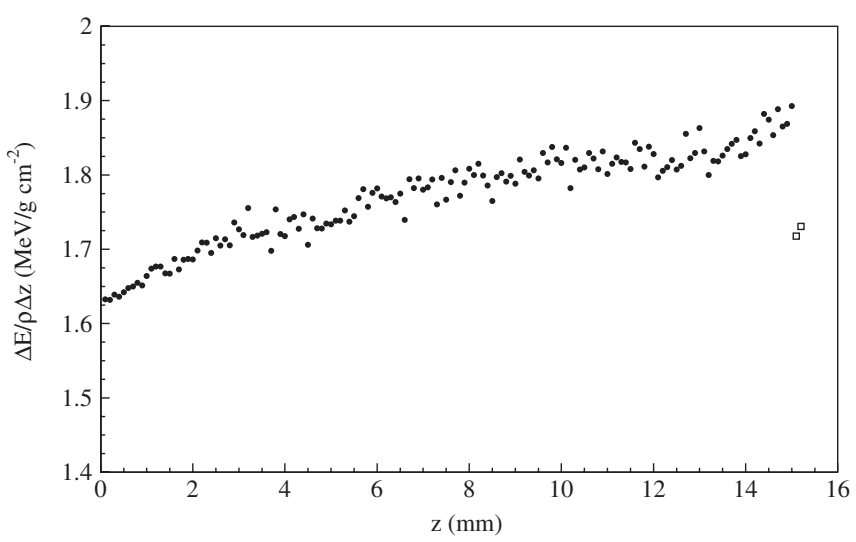

FIG. 21. Energy deposited by a $1.5 \mathrm{TeV}$ undisrupted beam (in $\mathrm{MeV} / \mathrm{g} \mathrm{cm}^{-2}$ ) along the exit window: the last two points correspond to the thin aluminum foil. 
TABLE VI. Mechanical and thermal properties of materials used in the exit window, instantaneous temperature increase, and cyclic thermal stress at the beam impact.

\begin{tabular}{lccccc}
\hline \hline Material & $E(\mathrm{GPa})$ & $\alpha\left(\mathrm{K}^{-1}\right)$ & $C(\mathrm{~J} / \mathrm{g} \mathrm{K})$ & $\Delta T(\mathrm{~K})$ & $\sigma_{c}(\mathrm{MPa})$ \\
\hline $\mathrm{C}-\mathrm{C}$ & 70 & $7 \times 10^{-6}$ & 0.53 & 3.0 & 1.5 \\
$\mathrm{Al}$ & 70 & $2.3 \times 10^{-5}$ & 0.90 & 1.7 & 2.7 \\
\hline \hline
\end{tabular}

Between two consecutive bunch trains (over a period $\tau=20 \mathrm{~ms}$ ), the heat produced by the impact of $n N_{b}$ particles is spread transversally with a typical diffusion length $\tilde{d}=\sqrt{k \tau / \rho C}$, where $k$ is the thermal conductivity. For the sake of simplicity, other cooling processes, such as the circulation of a gas on the window surface or thermal radiation, are not considered. In the carbon-carbon thick window and the aluminum foil, $k$ is, respectively, 0.24 and $2.37 \mathrm{~W} / \mathrm{K} \mathrm{cm}$, which leads to a diffusion length of the order of $1 \mathrm{~mm}$ in both materials. The exit window has a race-track shape, with a radius of $20 \mathrm{~cm}$ and a straight length of $54 \mathrm{~cm}$. The undisrupted beam hits this window $7.1 \mathrm{~cm}$ below the center of the upper half circle. The average distance from the beam spot to the edge of the window is $30 \mathrm{~cm}$, i.e., 300 times larger than the typical diffusion length. Therefore, the thermal equilibrium is reached after about $6 \mathrm{~s}$ of noncolliding beam operation.

For the sake of simplicity, we momentarily consider a circular symmetry for the exit window, in order to simplify the analytical calculations (one may indeed argue that the most relevant quantity to determine the equilibrium condition is the average distance between the beam impact point and the edge of the window, which determines how fast the heat is diffused). In cylindrical coordinates, the equilibrium temperature distribution is derived from the heat equation with no time dependence:

$$
-\frac{k}{r} \frac{\partial}{\partial r} r \frac{\partial T}{\partial r}=p(r) .
$$

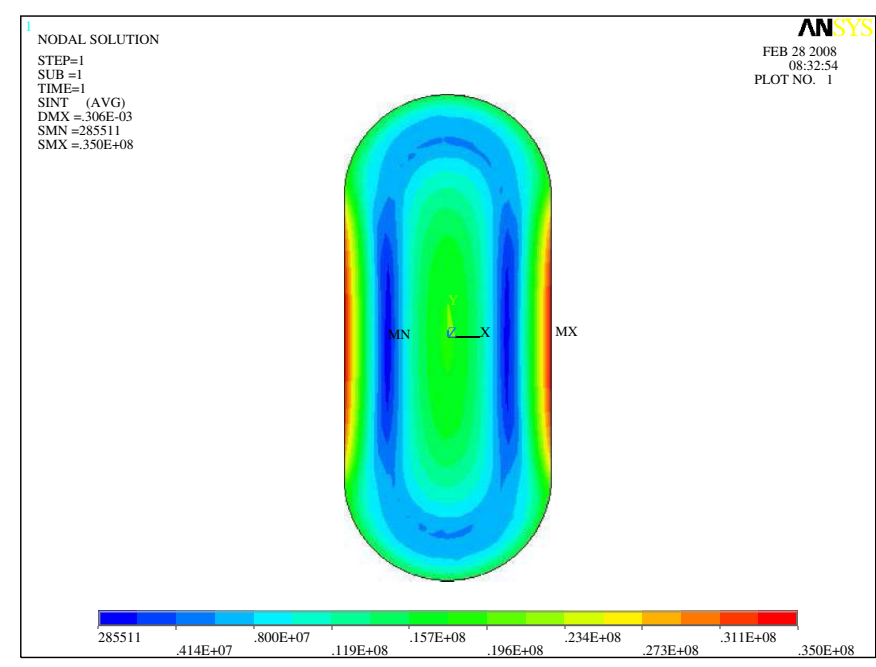

In this equation, $p(r)$ is the power distribution (per unit volume). In the following, we proceed as in [19] and we assume that the power distribution is

$$
p(r)=n N_{b} f \times\left(\frac{d E}{d x}\right) \times \frac{2 \sigma_{\text {beam }}^{2}}{\pi\left(r^{2}+2 \sigma_{\text {beam }}^{2}\right)^{2}} .
$$

It is similar to a Gaussian distribution, and it has the advantage that Eq. (12) can be solved analytically. At equilibrium, the temperature difference between the beam spot and the window edge is

$$
T(0)-T(R)=\left(\frac{d E}{d x}\right) \times \frac{n N_{b} f}{4 \pi k} \ln \left(1+\frac{R^{2}}{2 \sigma_{\text {beam }}^{2}}\right)
$$

Replacing $R$ by the average distance between the beam spot and the edge of the window $(30 \mathrm{~cm})$, we find that the equilibrium temperature difference is $76 \mathrm{~K}$ in the carboncarbon thick window and $14 \mathrm{~K}$ in the thin aluminum foil, therefore much smaller than the melting temperatures of these two materials.

In addition to the thermal constraints discussed above, the exit window must withstand the pressure difference between its two faces. The corresponding static stress $\sigma_{s}$ was computed with ANSYS [22], only for the carbon-carbon composite, since the aluminum thin leak-tight foil is fully supported by the thick window. Assuming an atmospheric pressure load of $0.1 \mathrm{MPa}$, uniformly distributed over the cross section of the window, as well as no degree of freedom around its circumference, our simulations yield a maximal mechanical stress of $35 \mathrm{MPa}$ and a displacement of $0.3 \mathrm{~mm}$ at the center of the window. The distribution of the stress intensity over the window is shown in Fig. 22. The largest stress is obtained on the lateral edges, where there is no thermal stress. In the beam impact region, $\sigma_{s}$ is 2-3 times smaller, around 10-15 MPa, which is much

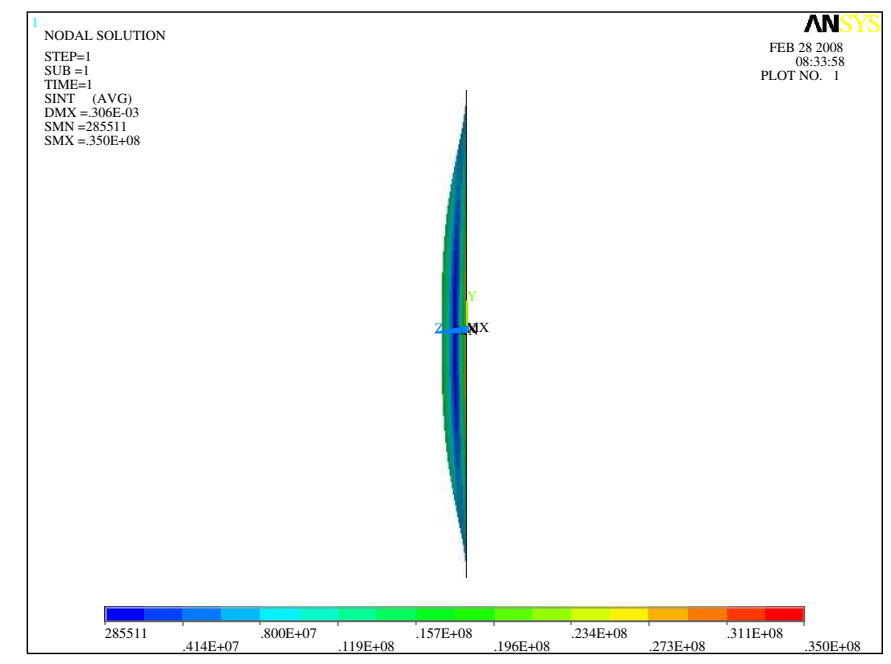

FIG. 22. (Color) Displacement and distribution of the mechanical stress intensity over the CLIC exit window. 
smaller than the tensile strength of the carbon-carbon composite (350 MPa typically).

Another exit window must be fabricated, for the dump of the wrong-sign charged particles. We also propose a $1.5 \mathrm{~cm}$ thick layer of carbon-carbon composite and a $0.2 \mathrm{~mm}$ thin foil of aluminum. This second exit window has an elliptical shape with an area of $0.35 \mathrm{~m}^{2}$, therefore we expect the same mechanical stress levels as in the $0.34 \mathrm{~m}^{2}$ exit window of the main beam. Moreover, the thermal stress is negligible because, whenever produced, the wrong-sign charged particles of the coherent pairs carry much less power than the main beam and are spread all over the window.

\section{IDEAS FOR POST-COLLISION BEAM MONITORING AT CLIC}

The primary goal of the CLIC post-collision line is to transport the outgoing beams to their dump with as small losses as possible. In addition, it should be used to measure the quality of the $e^{+} e^{-}$collisions. Here, we discuss the implementation of diagnostics along the CLIC postcollision line, in order to monitor the small beam-beam offsets that may occur at the interaction point during the tuning of the machine.

\section{A. Influence of small beam-beam offsets at the interaction point on the outgoing beams}

If the beam waists are not exactly at the same longitudinal position when electrons and positrons collide, then the two incoming bunches have different transverse dimensions and they experience different electromagnetic fields at the interaction point. Not only a luminosity loss occurs, but the amount of beamstrahlung photons and coherent pairs accompanying the two outgoing beams significantly differ, as illustrated in Fig. 23. Comparing the amount of secondary particles leaving the interaction point in each direction therefore gives information on the transverse aspect ratio between the colliding beams. For simplicity, both incoming bunches are simulated with the same properties (emittance, charge, length) and no additional offset is introduced here.

A comparison of the vertical beam profiles at the end of the post-collision lines is shown in Fig. 24, when one of the incoming beams is twice larger than the other (nominal) one at the interaction point, horizontally and vertically. The smaller incoming beam (2) experiences a weaker electromagnetic field than the larger incoming beam (1), and thus a less significant disruption during the bunch crossing. This can be clearly observed when comparing the outgoing beam distributions at the end of the two post-collision lines.

The luminosity, the disruption process, and, in turn, the amount of beamstrahlung photons and coherent pairs, are also significantly affected by vertical offsets in position
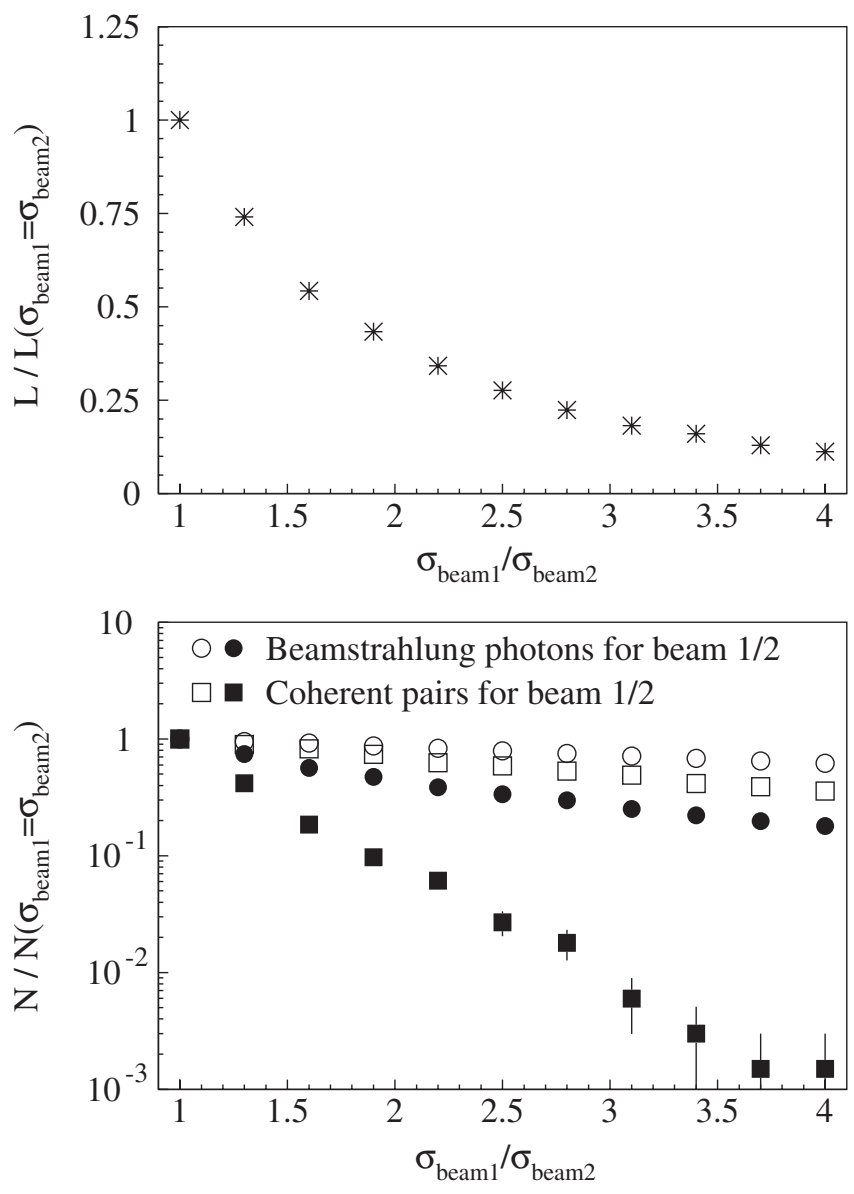

FIG. 23. Influence of a (common) transverse size difference between the incoming bunches on the luminosity (top) and on the amount of beamstrahlung photons and coherent pairs (bottom).

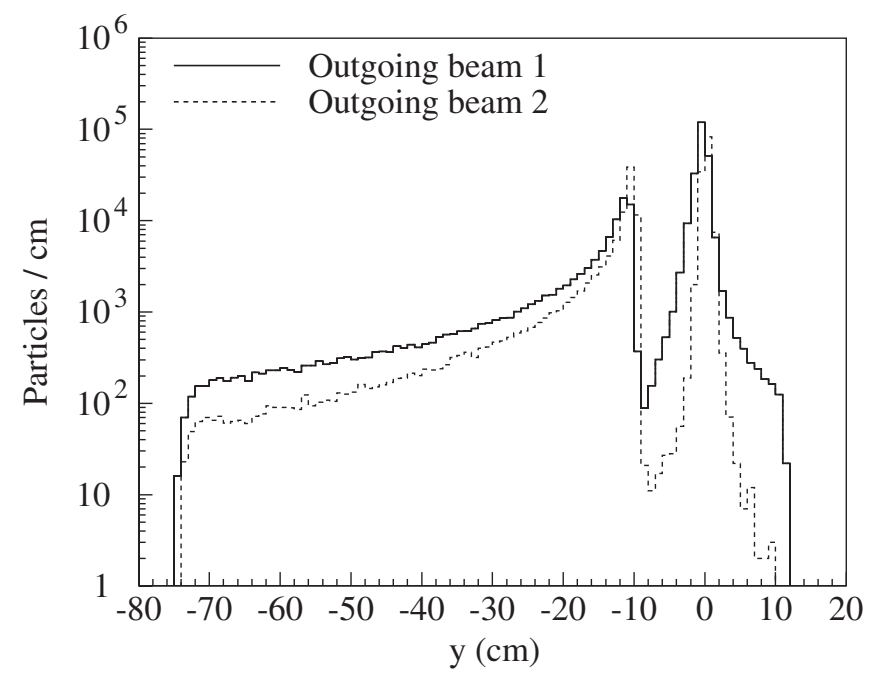

FIG. 24. Vertical profiles for the charged beams and the beamstrahlung photons at the end of each post-collision line, when the incoming beam 1 has twice the transverse dimensions of the incoming beam 2 at the interaction point. The distributions shown here correspond to $10^{5} e^{+} e^{-}$collisions, and not one bunch crossing. 
and angle (note that, since the incoming beams are flat, horizontal offsets are much less relevant).

For relatively small position offsets $\Delta y$, the electromagnetic field seen by the bulk of charged particles in one incoming beam increases with the distance to the other beam. So does the disruption and, in turn, the emission of beamstrahlung photons and coherent pairs. For larger position offsets, the field seen by each bunch becomes smaller and smaller as the distance between the beams increases. The strongest beam-beam effects are obtained when $\Delta y \simeq$ $8 \mathrm{~nm}$. Each incoming beam receives a kick towards the other beam when passing through its strong electromagnetic field. As a result, the $y^{\prime}$ distribution of the disrupted beam gets not only a large rms value, but also a nonzero mean value. The same conclusion is true for the coherent pairs. The particles that have the same charge as the disrupted beam receive a vertical kick in the same direction, but with a larger amplitude, because their energy is lower. For the particles of the coherent pairs with the wrong-sign charge, the vertical kick is in the opposite direction. Since the beamstrahlung photons do not carry any electric charge, the mean value of their $y^{\prime}$ distribution remains
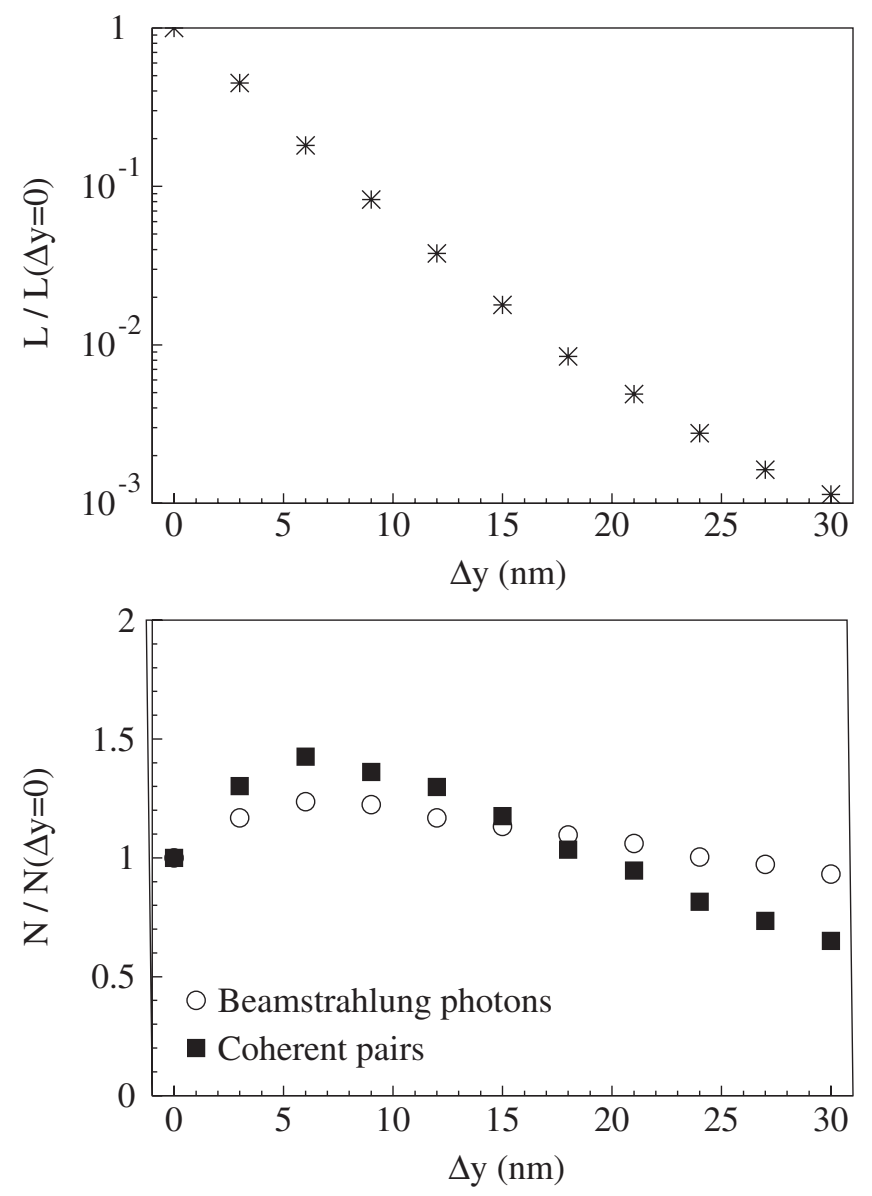

FIG. 25. Influence of a vertical position beam-beam offset on the luminosity (top) and on the amount of beamstrahlung photons and coherent pairs (bottom).
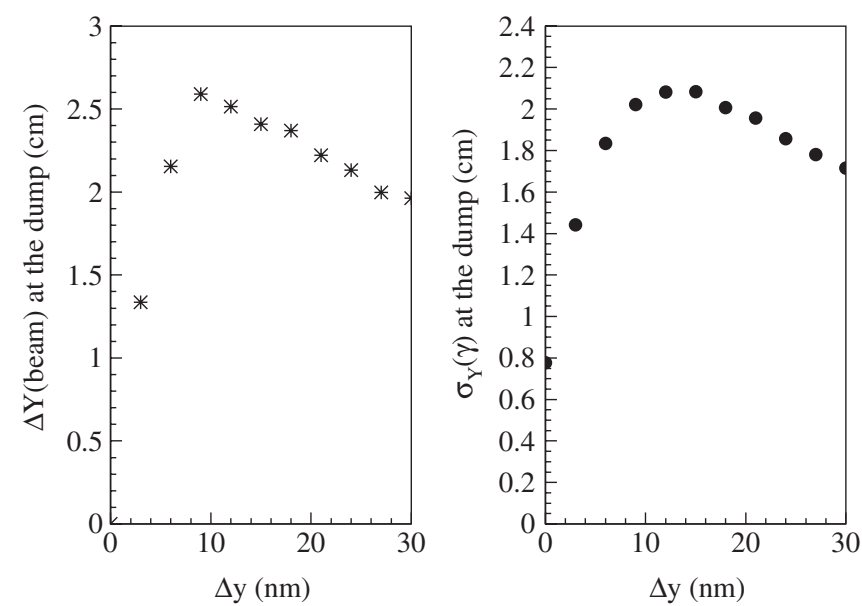

FIG. 26. Influence of a vertical position beam-beam offset on the position of the high-energy peak of the disrupted beam (left) and on the vertical size of the beamstrahlung photon cone (right).

equal to zero, but the associated rms value is significantly larger than in the case of ideal collisions. The dependence of the luminosity and of the number of beamstrahlung photons and coherent pairs on $\Delta y$ is shown in Fig. 25 (for simplicity, both incoming bunches have the same properties and no other offset is introduced). The variation with $\Delta y$ of the position of the high-energy peak and of the beamstrahlung photon cone size at the exit window are shown in Fig. 26. Note that the vertical displacement of the disrupted beam occurs in opposite directions for electrons and positrons when $\Delta y \neq 0$, while beamstrahlung photon cones are identical in both post-collision lines.

A small vertical angular offset $\Delta y^{\prime}$ at the interaction point may also affect the disruption process and thereby the number of secondary particles. Together with a measurement of the (vertical) profiles of the outgoing beams near their respective exit windows, a monitoring of the power transported by the beamstrahlung photons and the coherent pairs seems extremely useful to control the quality of the $e^{+} e^{-}$collisions. A detailed discussion of the CLIC machine tuning strategies is beyond the scope of this paper. However, in order to illustrate our statement, Fig. 27 shows that accurately monitoring the amount of beamstrahlung photons and coherent pairs in the post-collision lines, while (vertically) scanning one incoming beam through the other one at the interaction point, helps derive information regarding the quality of the $e^{+} e^{-}$collisions. Hence, it is important to design post-collision diagnostics aiming at measuring the secondary outgoing beams.

\section{B. Implementation of instrumentation devices along the CLIC post-collision line}

The CLIC post-collision line is an extremely hostile environment due to the high radiation levels that will destroy any device exposed directly to the beam, which implies that noninvasive diagnostics are needed. We dis- 

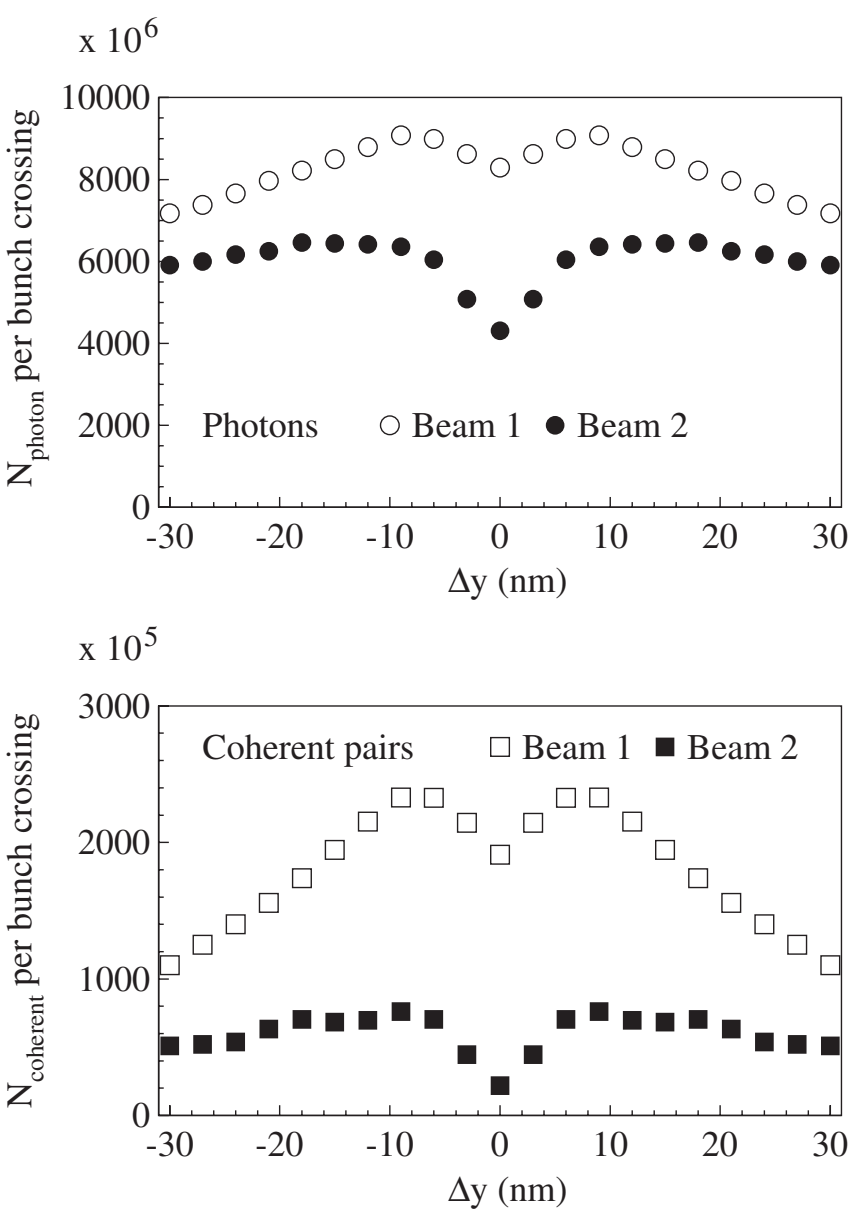

FIG. 27. Variation of the amount of beamstrahlung photons (top) and coherent pairs (bottom) when scanning one beam through the other at the interaction point, assuming that the incoming beam (1) has twice larger transverse dimensions than the incoming beam (2).

cuss a compilation of devices that could prove useful in extracting important information from the outgoing beams, by analyzing the temperature distribution in the dump, the image charges in the vacuum pipe, or various types of radiation emanating from the interaction point.

\section{Tail monitors}

When the beams are in collision, a very pronounced lowenergy tail is present in the energy spectrum of the outgoing main beams that travel along the post-collision line. These tails deposit a significant amount of energy on (and inside) the collimators sandwiched between the windowframe magnets. Hence, an increased amount of radiation at these strategic locations is correlated to the beamstrahlung parameter and is thus a clear signature for colliding beams. A high level of radiation is, of course, a problem for diagnostic devices which tend to malfunction after a long period of exposure. At both the proton and electron rings of HERA, this problem was solved by using radiation detec- tors based on PIN diodes in order to monitor the beam losses [23], albeit at a lower rate than anticipated at CLIC.

A possible layout for such a detector is based on reversebiased PIN diodes. Two diodes are coupled in series, in order to operate in coincidence, and should be wrapped in dark masking tape to avoid illumination by normal light. The diodes do not conduct when "dark" or unexposed to radiation, but do conduct when radiation hits them. The voltage drop over a resistor is fed into a unipolar op-amp that acts as an impedance converter to a $50 \Omega$ line.

Such a detector could be embedded in the body of the collimators located between the window-frame magnets. Shielding, for instance by the adjacent dipoles, is likely to protect the diodes from radiation generated elsewhere in the post-collision line, while they would be exposed to the spray of low-energy particles directly impacting on the collimator. A detailed study with GEANT [16] or a similar simulation program is needed to determine how much signal the PIN radiation monitors are exposed to upon impact of a particle on the collimator.

\section{Monitoring of the coherent pairs}

In the CLIC post-collision line, the wrong-sign charged particles of the coherent pairs are directed to their own dump. They can serve as a luminosity monitor since the beam-beam interaction is the only source of such wrongsign charged particles, apart from beam losses and synchrotron radiation that may produce $e^{+} e^{-}$pairs when hitting the vacuum pipe near the intermediate dump. This requires careful shielding or possibly a threshold detector for high-energy wrong-sign charged particles. Since the total power in the coherent pairs is much lower (about $1 \%$ $2 \%$ ) than in the primary beam, more advanced detection devices can be placed just upstream or in the intermediate beam dump. In particular, we suggest to use a device similar to a drift chamber, but more robust. The wires should be oriented horizontally to determine the vertical distribution of the wrong-sign charged particles at their dump. This provides information about the energy of the detected particles, because all of them emanate from a well-defined source point, the interaction point, see Fig. 11.

\section{Beamstrahlung monitor}

We consider a beamstrahlung monitor similar to the one used at the SLAC Linear Collider [24] but based on the detection of high-energy muons rather than electrons. Its layout is shown in Fig. 28.

Beamstrahlung photons impinging on the beam dump pass through the exit window and possibly a plate that converts them predominantly into $e^{+} e^{-}$and $\mu^{+} \mu^{-}$pairs. All shower products are absorbed, except most of the muons which can penetrate the dump enclosure. In a gas volume behind the dump, muons generate Cerenkov radiation that can be then picked up by a photomultiplier tube. 


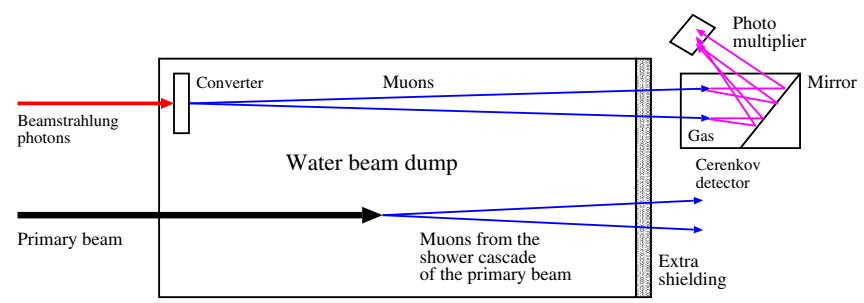

FIG. 28. (Color) Cerenkov beamstrahlung detector based on the conversion of high-energy beamstrahlung photons into muon pairs, that are the only charged particles penetrating the beam dump and can be detected by their emission of Cerenkov light in a gas detector. Note that the high-energy muons generated by the primary beam are predominantly forward peaked and miss the Cerenkov detector.

By judiciously choosing the parameters of the Cerenkov detector, one can discriminate the low-energy muons generated by the synchrotron radiation from the chicane dipoles, which has a critical energy $\epsilon_{c} \simeq 1.5 \mathrm{GeV}$. The energy of the beamstrahlung photons is considerably higher and lies above the Cerenkov threshold given by the refractive index of the gas.

The number of Cerenkov photons $N$ as a function of the incident beamstrahlung photon energy $\hbar \omega$ is given by the convolution of the muon creation probability with the Cerenkov photon generation probability. Here, we only briefly summarize the estimated signal strength, but we refer to [25] and the original publications about the SLC beamstrahlung detector [26-28] for more details. The conversion of photons into muon pairs is governed by the Bethe-Heitler cross section $\sigma_{\mathrm{BH}}$ [29], where the electron mass is replaced by the muon mass $m_{\mu}$. The probability to convert the incident photon into a muon pair is then $P=$ $1-e^{-n_{0} d \sigma_{\mathrm{BH}}} \simeq n_{0} d \sigma_{\mathrm{BH}}$, where $n_{0}$ and $d$ are the density and the thickness of the converter, respectively. The energy distribution of the muons is derived from the differential Bethe-Heitler cross section [29]. It is almost constant over the entire kinematically accessible range: the probability of finding a muon with any energy $\epsilon$ is simply $2 / \hbar \omega$. Once inside the gas volume of the Cerenkov detector with a length $l$, muons with an energy $\gamma_{\mu} m_{\mu} c^{2}$ generate the following number of Cerenkov photons per frequency [30]:

$$
\frac{d N}{d \nu} \simeq \frac{2 \pi \alpha l}{c}\left[1-\frac{1}{n^{2}}\left(1+\frac{1}{\gamma_{\mu}^{2}}\right)\right]
$$

The emission of Cerenkov light has a threshold given by $\gamma_{\mu}>\gamma_{0}=n / \sqrt{n^{2}-1}$. Using the same gas as in the SLC beamstrahlung detector (i.e. $n=1+2 \times 10^{-4}$ ) yields a threshold $\gamma_{0}=50$ (i.e. $5.3 \mathrm{GeV}$ ) for Cerenkov light emission by muons.

Using $\gamma_{B}=\hbar \omega / m_{\mu} c^{2}$ for notational convenience and assuming that the monitor detects Cerenkov photons within a frequency bandwidth $\Delta \nu=5 \times 10^{14} \mathrm{~Hz}$ (i.e. the entire range of the visible spectrum), the number of
Cerenkov photons $N$ is given by

$$
N(\hbar \omega) \simeq n_{0} d \sigma_{\mathrm{BH}} \frac{4 \pi \alpha l \Delta \nu}{c}\left(\frac{1}{\gamma_{0}}-\frac{1}{\gamma_{B}}\right)^{2} .
$$

Using a thickness $d=1 \mathrm{~mm}$ for the iron converter plate $\left(Z=26\right.$ and $\left.n_{0}=8.4 \times 10^{22} / \mathrm{cm}^{3}\right)$ and a length $l=1 \mathrm{~m}$ for the gas volume, we find

$$
N=0.18 \times\left(\frac{1}{50}-\frac{1}{\gamma_{B}}\right)^{2} \quad \text { if } \gamma_{B}>\gamma_{0} .
$$

As a result, we expect about $5 \times 10^{-5}$ Cerenkov photons per incident beamstrahlung photon with an energy significantly above the threshold of $5.3 \mathrm{GeV}$, say $50 \mathrm{GeV}$. For standard CLIC beam parameters, one expects about $8 \times$ $10^{9}$ beamstrahlung photons per bunch, of which most exceed the threshold energy. Hence, one expects about $4 \times$ $10^{5}$ Cerenkov photons per bunch, which should be reliably detectable with a photomultiplier. Detecting the current of the photomultiplier using fast analog digital converters with $\mathrm{GHz}$ bandwidth yields information about the disruption process at the interaction point, even within a bunch train which only lasts 156 ns. Also, we expect only a moderate background signal, because the muons that are generated by the shower cascade of the primary beam with enough energy to trigger the Cerenkov detector are strongly peaked forward. Muons with an energy below the Cerenkov threshold could more easily acquire sufficient transverse momentum to reach the Cerenkov detector, but they would not trigger it. This rough discussion needs to be refined with more thorough simulations, in order to optimize the parameters of this beamstrahlung monitor.

\section{Beam dump thermometry}

The power deposited by the outgoing beams in the main (water cooled) dump is about $14 \mathrm{MW}$. Of course, this huge power load heats the water and its temperature needs to be monitored for safety reasons. Since the extracted beam passes through a vertical chicane, it is widened vertically. Determining the vertical temperature distribution thus also yields some insight into the disrupted beam size on the target. We propose to use an interferometric setup, which is based on monitoring the change of the refractive index of water as a function of the temperature [31]. Indeed, the refractive index of water varies as $n=1.341-2.262 \times$ $10^{-5} T[\mathrm{~K}]$, when keeping all other parameters, such as pressure and wavelength, at standard values. Following [31] further and assuming that the temperature distribution along the one arm of the interferometer follows a Gaussian temperature distribution $T(x)=T_{0} e^{-x^{2} / 2 \sigma^{2}}$ with a peak temperature $T_{0}$ above the average water temperature and a rms width $\sigma$, we find that the relative change in phase is given by 


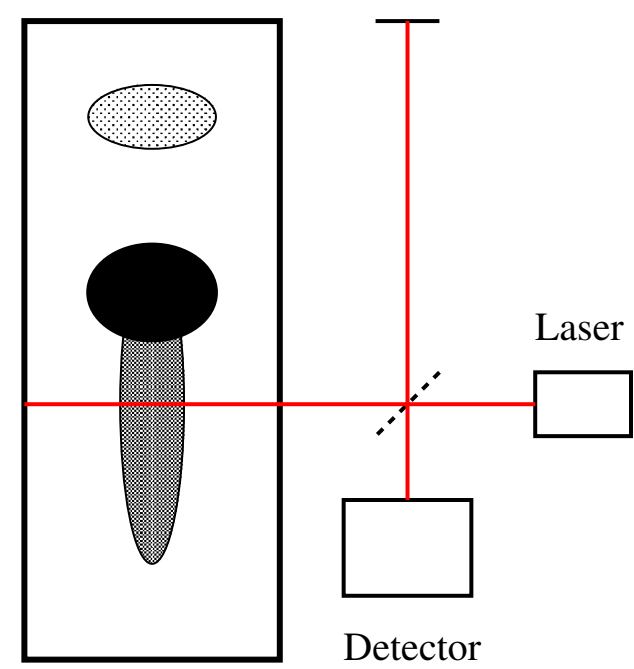

FIG. 29. (Color) Schematic layout of the interferometric thermometer proposed to measure temperature variations following energy deposition by the disrupted beam in the water dump.

$$
\Delta m=\frac{1}{\lambda} \int \Delta n(x) d x \simeq 96.3 \sigma T_{0},
$$

where $\lambda$ is the wavelength of light. A temperature change $T_{0}$ of the order of $1 \mathrm{~K}$ and a $\sigma$ of the order of $1 \mathrm{~cm}$ result in changes of $m$ that are of the order of unity.

As illustrated in Fig. 29, we suggest to place one arm of the interferometer horizontally across the dump, at a vertical position below the position where the main beam impinges. This requires installation of a window at the side of the water dump, where the light enters, as well as a mirror inside the dump, which allows monitoring relative changes of the temperature, provided that turbulences do not interfere adversely. This must be carefully investigated for a complete design of this diagnostic device.

\section{CONCLUSION}

This paper presents a conceptual design of the postcollision line for the CLIC machine. It first separates the various components of the outgoing beam in compact extraction magnets, which provide a total bending angle of $2.56 \mathrm{mrad}$ at $1.5 \mathrm{TeV}$. Charged particles with $\delta p / p<$ -0.95 are absorbed in collimators, which ensures that the beam transport through the extraction magnets remains loss free. After their physical separation from the other outgoing beams, the particles of the coherent pairs with the wrong-sign charge are immediately brought to their dump. As for the main disrupted beam and the beamstrahlung photons, they are transported inside the same vacuum pipe towards a common dump. The bend provided by the window-frame magnets is followed by a bend in the opposite direction, provided by four C-type dipole magnets. All beamstrahlung photons and the charged particles with $\delta p / p>-0.84$ pass through the vertical chicane and reach the dump, $50 \mathrm{~m}$ downstream. The lost particles are absorbed in the intermediate dump upstream of the first Ctype magnet.

Analytical calculations and numerical simulations of the exit window, to be placed $150 \mathrm{~m}$ downstream of the interaction point in our design, showed that both the temperature increase and the thermal stress at the (undisrupted) beam impact point remain reasonable. The mechanical stress was also computed, and found to be about 1 order of magnitude smaller than the tensile strength of the carbon-carbon composite used for the fabrication of the window.

An accurate analysis of the transverse beam profiles at the end of the CLIC post-collision line (or on the face of the intermediate dump for the wrong-sign charged particles of the coherent pairs) allows to derive some relevant information on the $e^{+} e^{-}$collisions. In particular, small vertical beam-to-beam offsets in position and/or angle, which affect the disruption process, can be identified by measuring the displacement and/or the distorsion of the outgoing beams. Monitoring the flux of beamstrahlung photons and coherent pairs is also crucial to identify beam-tobeam offsets, and thereby to control the quality of the $e^{+} e^{-}$collisions. In this paper, we have proposed four post-collision diagnostics tools aimed at monitoring the disruption process: tail monitors that are embedded in the collimators, measurement of the flux and vertical profile of the wrong-sign charged particles of the $e^{+} e^{-}$coherent pairs at the intermediate dump, beamstrahlung monitor based on the detection of the Cerenkov light emitted by muons behind the main dump, and an interferometric setup to monitor water temperature profiles in the dump.

Our design of the CLIC post-collision line is entirely based on DIMAD particle tracking studies. This program allows one to follow a large number of electrons, positrons, and beamstrahlung photons, without using large amounts of memory and/or computing time. However, it does not simulate the interactions of lost particles with the surrounding matter, once they have left the vacuum pipe or after hitting a collimator. For a complete study of the performance of the post-collision line, BDSIM simulations will be needed, in particular, in order to estimate the background due to backscattered particles (photons and neutrons) at the interaction point.

Other critical issues to be addressed in the future are an estimation of the activation levels near the collimators and the intermediate dump, together with a study of the stray radiation into the neighboring beam line sections. Also, an accurate analysis of the impact of synchrotron radiation on the losses in the magnets and on the backscattered flux of secondary particles must be performed. A more detailed design of the magnetic elements, collimators, and dumps will be needed as well, taking into account technical and economical considerations such as power consumption, cooling, and constraints arising from the large aperture vacuum chambers. The integration of the new diagnostics 
tools proposed in this manuscript into the CLIC postcollision line must also be addressed in future, more technical, iterations of the design.

\section{ACKNOWLEDGMENTS}

The authors wish to thank T. Zickler and K. Elsener for their advice on magnet technology and design, J. Vollaire and M. Lantz for their help with FLUKA, and B. Goddard, $\mathrm{R}$. Veness, and W. Weterings for their comments on the exit window and their help with ANSYS. This work is supported by the Commission of the European Communities under the 6th Framework Program "Structuring the European Research Area", Contract No. RIDS-011899.

[1] I. Wilson, Phys. Rep. 403, 365 (2004).

[2] D. Schulte and F. Zimmermann, CLIC note 484.

[3] R. Arnold, K. Moffeit, Y. Nosochkov, W. Oliver, A. Seryi, E. Torrence, and M. Woods, in Proceedings of the 21 st Particle Accelerator Conference, Knoxville, 2005 (IEEE, Piscataway, NJ, 2005).

[4] A. Ferrari, CLIC note 662.

[5] S. Darbha, SLAC-TN-07-013.

[6] H. Braun et al., CLIC note 764.

[7] D. Schulte, TESLA-97-08.

[8] P. Chen and K. Yokoya, KEK-91-002.

[9] P. Bambade, K. Mönig, C. Rimbault, and D. Schulte, Phys. Rev. ST Accel. Beams 9, 034402 (2006).

[10] D. Schulte, in Proceedings of LINAC'04, Lübeck, Germany.

[11] ILC Reference Design Report, Vol. 3 (2007), p. 191.
[12] H. Grote and F. Schmidt, in Proceedings of the 20th Particle Accelerator Conference, Portland, OR, 2003 (IEEE, New York, 2003).

[13] http://www.slac.stanford.edu/accel/ilc/codes/dimad.

[14] K. Brown, D. Carey, C. Iselin, and F. Rothacker, CERN 80-04.

[15] http://flc.pp.rhul.ac.uk/bdsim.html.

[16] S. Agostinelli et al., Nucl. Instrum. Methods Phys. Res., Sect. A 506, 250 (2003).

[17] P. Bambade and O. Dadoun, in Proceedings of the 2007 Particle Accelerator Conference, Albuquerque, New Mexico, 2007 (IEEE, Albuquerque, New Mexico, 2007).

[18] R. Veness, B. Goddard, S. Mathot, A. Presland, and L. Massidda, in Proceedings of the 10th European Particle Accelerator Conference, Edinburgh, Scotland, 2006 (EPSAG, Edinburgh, Scotland, 2006).

[19] M. Seidel, DESY-TESLA 95-18.

[20] A. Fasso, A. Ferrari, J. Ranft, and P. Sala, CERN-2005010, INFN/TC_05/11, SLAC-R-773.

[21] A. Fasso et al., in Proceedings of CHEP2003, La Jolla, CA.

[22] http://www.ansys.com.

[23] K. Wittenburg, DESY-HERA-2000-003.

[24] R. Field, Nucl. Instrum. Methods Phys. Res., Sect. A 265 , 167 (1988).

[25] V. Ziemann, CLIC note 736.

[26] E. Gero, Ph.D. thesis, University of Michigan, 1991 [UMI Report No. UMI-91-24010].

[27] V. Ziemann, SLAC-CN-379.

[28] V. Ziemann, SLAC-PUB-5595.

[29] L. Landau and E. Lifschitz, Relativische Quantentheorie (Akademie Verlag, Berlin, 1980).

[30] W.-M. Yao et al., J. Phys. G 33, 1 (2006).

[31] V. Ziemann, Nucl. Instrum. Methods Phys. Res., Sect. A 564, 587 (2006). 